BARBARA DO CARMO DOS SANTOS MARTINS

\title{
Avaliação da qualidade de vida na hipertensão arterial pulmonar
}

Tese apresentada à Faculdade de Medicina da Universidade de São Paulo para obtenção do título de Doutor em Ciências

Área de concentração: Pneumologia Orientador: Prof. Dr. Rogério de Souza

São Paulo 2008 
BARBARA DO CARMO DOS SANTOS MARTINS

\section{Avaliação da qualidade de vida na}

\section{hipertensão arterial pulmonar}

Tese apresentada à Faculdade de Medicina da Universidade de São Paulo para obtenção do título de Doutor em Ciências.

Área de concentração: Pneumologia

Orientador: Prof. Dr. Rogério de Souza

São Paulo

2008 


\section{Dedicatória}


A minha mãe, Ione, seu amor incondicional me dá confiança para enfrentar os desafios da vida.

A minha avó, Elisa, meu exemplo de amor, dedicação e força.

Aos meus afilhados, Grazziela, Bruna e André, presentes de Deus que me foram dados. 
Agradecimentos 
Definitivamente não foi fácil chegar ao momento de escrever o agradecimento, mas sem dúvida é muito gratificante, principalmente, por perceber o quanto as pessoas mais especiais na minha vida confiam no meu potencial e me ajudam a crescer.

Em primeiro lugar agradeço ao Rogério, pela oportunidade de realizar esse trabalho, por ter me dito muitas vezes: "Você não acredita no seu potencial, mas eu acredito!" e por ter me mostrado até onde posso chegar.

À mãe Ione, por estar sempre ao meu lado, sem medir paciência, esforço e apoio.

Ao meu pai, Antonio Carlos, que sempre me proporcionou condições para eu poder realizar minhas escolhas.

À Renata, minha irmã, meu carma, pela amizade singular que me dá força, coloca meus pés na realidade, dá colo e é capaz de ouvir 50 vezes a mesma aula para me dar confiança. À Família Romaguera (Re, Dani e André), a minha família de coração, por todo o apoio. A toda a minha família, Martins, Varlese, Thomazzi e Santos, pela torcida constante e pela imagem maravilhosa que vocês tem de mim.

À Alexandra, sempre disposta a ajudar, entender meus momentos, e principalmente, pelo apoio à realização desse trabalho. Sem dúvida, você fez parte dele!

A todos meus amigos da equipe de fisioterapia do HUUSP, que agüentaram meu horários loucos, meus momentos desesperados e me ajudaram sempre. E também aos amigos da equipe médica e de enfermagem da UTI - HUUSP pelo apoio

Ao Prof. Dr. Paulo Andrade Lotufo e Prof. Dr. Luis Marcelo Inaco Cirino pelo estímulo e investimento no meu crescimento profissional, espero conseguir retribuir toda a ajuda que vcs me deram!

À Viviane com quem dividi todo esse processo, desde as mais difíceis provas e aulas até ótimas viagens para colher a recompensa dos nossos trabalhos. 
Ao Grupo de Circulação Pulmonar, Jardim, André, Caio, Susana, Ana Paula, simplesmente porque esse trabalho é nosso!!!

Ao Norberto e ao Karluz pela ajuda nos momentos difíceis, me mostrando a melhor saída e pela confiança na minha capacidade. E a todo o grupo pela torcida.

Ao Chuahy, pela sua paciência que lhe é peculiar, me ajudou desde a primeira tabela até a última.

Aos meus amigos que estão sempre do meu lado, Patrícia, Pablo, Juliana, Graziela, Márcia. À Solve, tentando me mostrar que nada é tão terrível.

Às meninas do Protocolo, sempre dispostas a ajudar.

Aos pacientes de hipertensão pulmonar que não desistem da luta por uma melhor qualidade de vida.

E a Deus por me presentear com pessoas tão especiais, me dando condições de conquistar meus objetivos!

Obrigada! 
Sumário 
Resumo

Summary

1. INTRODUÇÃO 2

1.1. Hipertensão Pulomonar 2

1.2. Hipertensão Arterial Pulmonar 4

$\begin{array}{ll}\text { 1.3. Marcadores de resposta } & 8\end{array}$

1.3.1. Marcadores hemodinâmicos 8

1.3.2. Marcadores de imagem 9

1.3.3. Marcadores bioquímicos 9

1.3.4. Marcadores funcionais 10

1.3.5. Avaliação da qualidade de vida 12

2. OBJETIVOS 19

3. MÉTODOS 21

3.1. População 21

3.2. Critérios diagnósticos 21

3.3. Avaliação clínica e funcional 23

3.4. Análise estatística $\quad 25$

4. RESULTADOS 28

5. DISCUSSÃO 43

6. CONCLUSÃO

7. ANEXOS 53

8. REFERÊNCIAS 56

9. APÊNDICES $\quad 67$ 
Resumo 
A hipertensão arterial pulmonar é caracterizada pela elevação anormal dos níveis pressóricos do território arterial pulmonar com conseqüente falência da função ventricular direita. A doença é altamente incapacitante, conforme demonstrado pela limitação nos testes de avaliação funcional, como o teste de caminhada de seis minutos. Entretanto, o impacto da doença na qualidade de vida dos pacientes não é totalmente conhecido. O objetivo desse estudo foi avaliar a qualidade de vida de pacientes com HAP através do questionário genérico SF-36, correlacionando os achados com os demais marcadores de gravidade da doença, assim como com a sobrevida. Os resultados mostraram que a QV apresenta forte correlação com TC6M e Classe Funcional e tem melhora significativa após tratamento específico. O Componente Físico do SF-36 teve significante associação com a sobrevida, permitindo concluir que a avaliação de qualidade de vida deve fazer parte da avaliação rotineira de pacientes com HAP.

Descritores: hipertensão pulmonar, qualidade de vida, caminhada, teste de exercício, sobrevida. 
Summary 
Pulmonary arterial hypertension $(\mathrm{PAH})$ is a rare but insidious disease characterized by progressive pulmonary vascular remodelling with increased pulmonary vascular resistance ultimately leading to right ventricular failure e progressive dyspnea, leading to profound functional limitations, how is demonstrated by the six minute walk test. However, there is limited information about quality of life (QoL) in patients with $\mathrm{PAH}$. The aim of this study was to evaluate the quality of life of PAH patients with the SF-36 generic questionnaire and compare the scores with the surrogate markers and identify the prognostic implication of this assessment. The results showed there was a good correlation with six minute walk test and functional class and there was a significant improvement in QoL after specific treatment. The physical component summary (PCS) of the SF36 QoLQ was significantly related to survival. Conclusion is that QoL is a useful tool for routine evaluation of $\mathrm{PAH}$ patients once it correlates with other surrogate markers, reflects treatment effect and predicts survival.

Descriptors: hypertension pulmonary, quality of life, walking, exercise test, survivorship. 


\section{Introdução}




\subsection{Hipertensão Pulmonar}

A hipertensão pulmonar é uma situação clínica comum a diversas doenças, caracterizada pela elevação anormal dos níveis pressóricos do território vascular pulmonar. Esse conjunto de doenças pode compartilhar diversos achados anatomopatológicos, porém com significativas diferenças fisiopatológicas e prognósticas. ${ }^{1,2}$

O grande avanço que se seguiu ao maior conhecimento sobre os fenômenos fisiopatológicos relacionados à hipertensão pulmonar permitiu que fossem desenvolvidos novos medicamentos, tornando necessário classificar melhor a hipertensão pulmonar, visando a correta intervenção terapêutica e definição prognóstica.

Quando se confirma a presença de hipertensão pulmonar é necessário estabelecer quais os mecanismos ou condições associadas que levaram ao desenvolvimento dessa elevação pressórica. Em 2003, durante a Terceira Conferência Mundial de Hipertensão Pulmonar, realizada em Veneza (Itália), a classificação foi revisada e adquiriu o formato atualmente utilizado. Esta nova classificação é mais conveniente para subdividir os pacientes em função dos mecanismos fisiopatológicos envolvidos, do prognóstico e também da resposta terapêutica esperada. (Quadro. 1) ${ }^{3}$ 
Quadro. 1 - Classificação da hipertensão pulmonar, Veneza, 2003

1. Hipertensão arterial pulmonar

1.1- Idiopática

1.2- Familiar

1.3- Associada a:

1.3-1. Doenças vasculares do colágeno

1.3-2. Shunts sistêmico-pulmonares congênitos

1.3-3. Hipertensão portal

1.3-4. Infecção pelo HIV

1.3-5. Drogas/toxinas

1.3-6. Outras (tireodopatias, telangectasia familiar hereditária, hemoglobinopatias, doença de Gaucher, doenças mieloproliferativas, esplenectomia)

1.4 - Associada a acometimento capilar/venoso significativo

1.4-1. Doença pulmonar veno-oclusiva

1.4-2. Hemangiomatose capilar pulmonar

1.5 - Hipertensão persistente do recém-nascido

2. Hipertensão venosa pulmonar

2.1 - Cardiopatia de câmaras esquerdas

2.2 - Valvopatias à esquerda

3. Hipertensão pulmonar associada a pneumopatias elou hipoxemias

3.1 - Doença Pulmonar Obstrutiva Crônica

3.2 - Pneumopatia intersticial

3.3 - Doenças respiratórias relacionadas ao sono

3.4 - Hipoventilação alveolar

3.5 - Exposição crônica a altas altitudes

3.6 - Anormalidades do desenvolvimento

4. Hipertensão pulmonar devido à doença embólica elou trombótica crônica

4.1 - Obstrução tromboembólica das artérias pulmonares proximais

4.2 - Obstrução das artérias pulmonares distais

4.3 - Embolia pulmonar não-trombótica (tumor, parasitas, material estranho)

5. Miscelânia

Sarcoidose, histiocitose $X$, linfangioleiomiomatose, compressão dos vasos pulmonares (adenopatia, tumor, mediastinite fibrosante) 


\subsection{Hipertensão arterial pulmonar (HAP)}

A hipertensão arterial pulmonar é uma doença caracterizada pelo acometimento dos ramos arteriais periféricos da circulação pulmonar, levando ao aumento da resistência vascular pulmonar e à disfunção ventricular direita. ${ }^{1}$ De uma forma geral, a HAP pode estar associada a um grande número de condições clínicas ou ainda ser resultado do acometimento vascular pulmonar isolado, caracterizando a hipertensão arterial pulmonar idiopática (HAPI). ${ }^{4}$

Define-se como hipertensão arterial pulmonar a presença de pressão média de artéria pulmonar $\geq 25 \mathrm{mmHg}$ no repouso ou $\geq 30 \mathrm{mmHg}$ durante 0 esforço, com valores normais de pressão de oclusão da artéria pulmonar $(<15 \mathrm{mmHg}){ }^{5,6}$

O termo hipertensão arterial pulmonar idiopática (HAPI) é usado para descrever os quadros em que o fator causal relacionado à hipertensão não é identificado, aceitando-se, então, um acometimento primário do território arterial pulmonar. ${ }^{1,7,8}$

A HAPI é uma doença rara, com etiologia desconhecida e incidência estimada de 1 a 2 casos por milhão por ano e prevalência de cerca de 15 casos por milhão de habitantes, sendo mais comum em mulheres (aproximadamente 2:1), com idade média ao diagnóstico em torno de 30 a 35 anos e, se não tratada, com sobrevida média de menos de 2,8 anos desde o diagnóstico. ${ }^{5,6}$

O processo de remodelação vascular, caracterizado pela hipertrofia da camada média e proliferação endotelial, reduz o fluxo sangüíneo, 
aumenta a pressão arterial pulmonar média (mPAP) e a resistência vascular pulmonar (RVP), levando ao aumento do trabalho ventricular direito e à hipertrofia cardíaca. A disfunção e insuficiência ventricular direita resultam da inabilidade em compensar estes efeitos.

Os sintomas dos pacientes com HAP são inespecíficos. A dispnéia relacionada aos esforços é o sintoma mais precoce e freqüente, apresentando caráter progressivo e refletindo a incapacidade do sistema cardiovascular em aumentar o débito cardíaco no esforço. Outros sintomas que podem estar presentes são: fadiga, pré-síncope, dor torácica e palpitações. ${ }^{16}$

Essa falta de especificidade na apresentação clínica faz com que o diagnóstico seja feito de forma tardia, podendo levar cerca de 2 anos o tempo entre o início dos sintomas e a confirmação diagnóstica. ${ }^{9}$

O processo diagnóstico é complexo, pois exige a investigação ativa de todas as condições associadas, como por exemplo, doenças do tecido conectivo, hipertensão porto-pulmonar, cardiopatias congênitas ou ainda doenças do parênquima pulmonar. O diagnóstico de HAPI só pode ser estabelecido após a exclusão de todas as possíveis causas associadas à gênese da hipertensão pulmonar.

Vários fluxogramas foram sugeridos para a investigação da hipertensão pulmonar, como o apresentado na figura 1, sugerido pelas diretrizes brasileiras para o manejo da hipertensão pulmonar. ${ }^{6}$ 


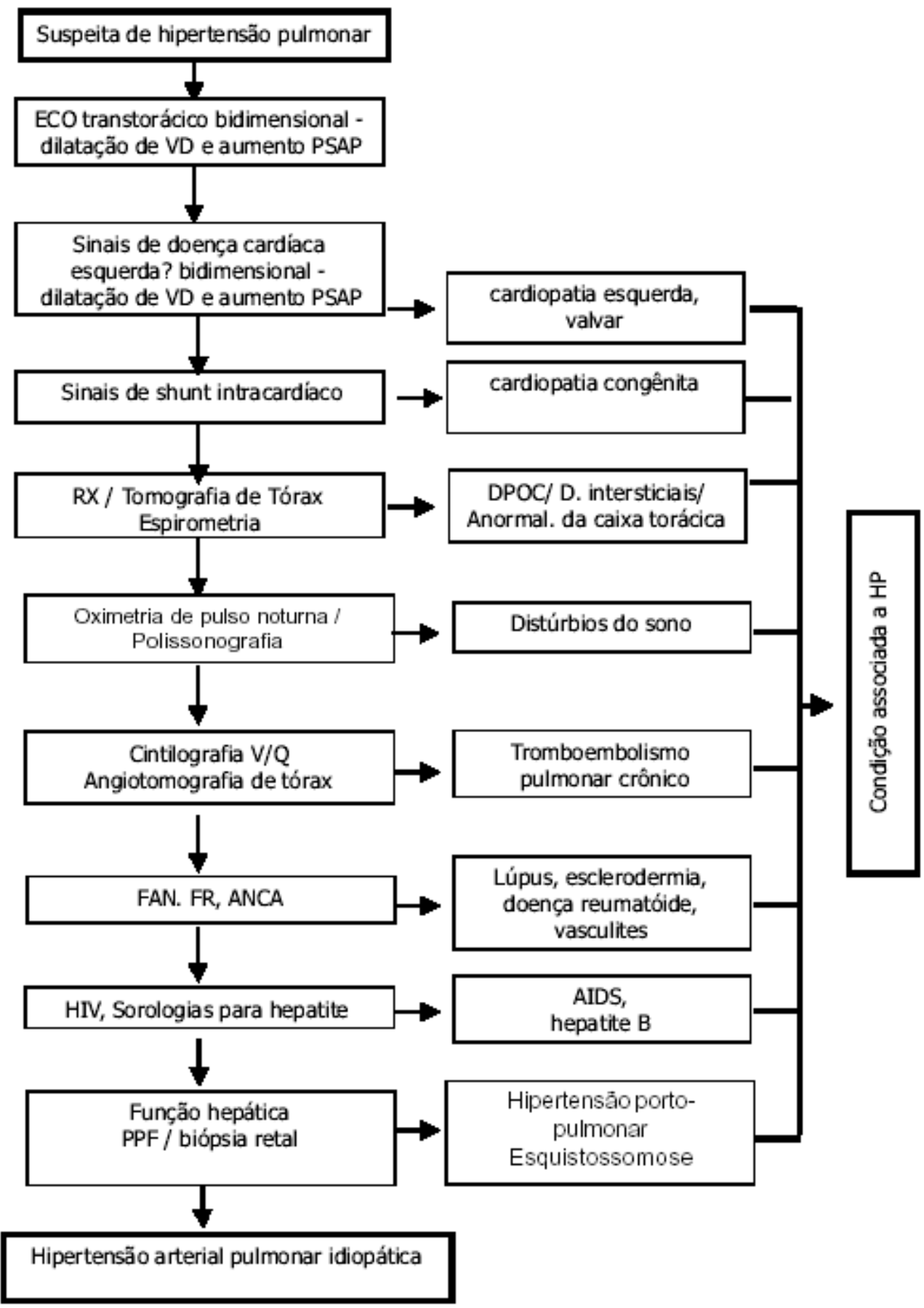

Figura 1. - Algoritmo diagnóstico nos pacientes com suspeita de Hipertensão Pulmonar. ${ }^{6}$ 
Após essa extensa investigação, tem-se ainda a necessidade de realizar um cateterismo cardíaco direito, única forma de assegurar a presença de hipertensão pulmonar, permitindo ainda a localização do território mais acometido com maior precisão (arterial ou venoso, de acordo com a mensuração da pressão de oclusão da artéria pulmonar) e a realização de testes agudos para avaliar a vaso-reatividade vascular pulmonar. 610

O aumento do conhecimento das diversas vias fisiopatológicas envolvidas nesse processo favoreceu o desenvolvimento de algumas opções atuais para o tratamento específico da hipertensão pulmonar.

A utilização de drogas com ação predominante sobre a circulação pulmonar é a base do tratamento específico. O tratamento específico é feito através do uso de bloqueadores dos canais de cálcio, em pacientes que apresentam a vasoconstrição como principal mecanismo envolvido na gênese da hipertensão pulmonar (reconhecidos pelo teste agudo com vasodilatador realizado durante o cateterismo cardíaco) ${ }^{11}$, prostanóides (epoprostenol ${ }^{12}$, iloprost ${ }^{13}$ ), antagonistas dos receptores de endotelina (bosentan, sitaxsentan, ambrisentan), ${ }^{14,15}$ inibidores da fosfodiesterase do tipo $\mathrm{V}$ (Sidenafil ${ }^{16}$, Tadalafil ${ }^{17}$ ) ou ainda, através do tratamento cirúrgico (tromboendarterectomia, septostomia ou transplante pulmonar) ${ }^{6}$. 


\subsection{Marcadores de resposta}

Em uma fase de desenvolvimento de novas modalidades terapêuticas, a avaliação da efetividade do tratamento é de fundamental importância. Essa avaliação deve ser feita através de marcadores que possuam sensibilidade e especificidade suficientes para refletir a resposta à intervenção. Os marcadores ideais de resposta têm que refletir os fenômenos fisiopatológicos envolvidos, possibilitar a extrapolação para respostas mais significativas (sobrevida, por exemplo) e a estratificação da gravidade da doença, com alta reprodutibilidade, pouca subjetividade e fácil emprego. ${ }^{18}$

Especificamente em HAPI, os marcadores mais utilizados nos estudos clínicos podem ser agrupados da seguinte forma: marcadores funcionais (classe funcional, teste de caminhada de seis minutos, teste cardiorrespiratório), marcadores hemodinâmicos, marcadores de imagem (ecocardiografia, ressonância nuclear magnética cardíaca), marcadores bioquímicos (ácido úrico, troponina, peptídeos natriuréticos) e avaliação da qualidade de vida. ${ }^{6}$

\subsubsection{Marcadores hemodinâmicos}

A hemodinâmica está profundamente alterada na HAP, com aumento acentuado dos níveis de pressão da artéria pulmonar, elevação da pressão do átrio direito e redução do débito cardíaco. ${ }^{4}$

A medida das variáveis hemodinâmicas através do cateterismo cardíaco direito tem correlação com prognóstico de pacientes com HAP. ${ }^{1,19}$ 
É um procedimento invasivo, mas necessário para confirmação diagnóstica e para a avaliação da resposta aguda frente à administração de vasodilatador de curta duração. 5,10

\subsubsection{Marcadores de imagem}

O ecocardiograma é utilizado rotineiramente no manuseio de pacientes com hipertensão pulmonar, tanto na fase diagnóstica quanto na avaliação prognóstica ${ }^{6}$. Entretanto, os achados relacionados ao prognóstico da HAP têm caráter tardio, pois refletem fases mais avançadas da doença, tais como: aumento das câmaras cardíacas, presença de derrame pericárdico, presença de disfunção ventricular direita e alterações nos volumes de enchimento do ventrículo esquerdo.

A ressonância magnética, com sua capacidade de definição anatômica do sistema cardiovascular, ainda é utilizada de modo experimental, mas traz grandes expectativas devido aos resultados iniciais na avaliação da resposta aguda e da resposta a intervenções terapêuticas em HAPI. ${ }^{20} 21$

\subsubsection{Marcadores bioquímicos}

Vários marcadores bioquímicos como a endotelina 1 , o dímero $D$, os peptídeos natriuréticos (BNP e proBNP), a troponina e o ácido úrico, foram estudados tanto para avaliar o prognóstico quanto a resposta terapêutica em pacientes com hipertensão pulmonar. ${ }^{6}$ 
O ácido úrico em níveis aumentados tem correlação com algumas variáveis hemodinâmicas, e os níveis basais tem valor prognóstico em relação à sobrevida. Porém, o grande número de interferentes no nível sérico de ácido úrico limita sua utilização no seguimento de pacientes.

Os níveis séricos de troponina T também mostraram correlação com o prognóstico de pacientes com HAPI, mas sua sensibilidade parece ser baixa quando a doença não se encontra em estágio avançado. ${ }^{22}$

Os níveis de peptídeos natriuréticos correlacionaram-se com classe funcional, com as medidas hemodinâmicas e com a resposta ao tratamento e sendo também, preditores independentes de sobrevida. ${ }^{23,24} 2526,27$

\subsubsection{Marcadores funcionais}

Um dos marcadores funcionais de gravidade utilizado em HAP é Classe Funcional da New York Heart Association / Organização Mundial da Saúde, modificada para HP.(Quadro. 2) ${ }^{3,6}$. É de fácil utilização, tem correlação com sobrevida e com dados hemodinâmicos ${ }^{19,28}$ 
Quadro. 2 - Classificação funcional da New York Heart Association modificada para HPI Organização Mundial de Saúde

CLASSE I: pacientes com HP, mas sem limitação das atividades físicas. Atividades físicas habituais não causam dispnéia ou fadiga excessiva, dor torácica ou síncope.

CLASSE II: pacientes com HP resultando em discreta limitação das atividades físicas. Estes pacientes estão confortáveis ao repouso, mas atividades físicas habituais causam dispnéia ou fadiga excessiva, dor torácica ou pré-síncope.

CLASSE III: pacientes com HP resultando em importante limitação das atividades físicas. Estes pacientes estão confortáveis ao repouso, mas esforços menores que as atividades físicas habituais causam dispnéia ou fadiga excessiva, dor torácica ou présíncope.

CLASSE IV: pacientes com HP resultando em incapacidade para realizar qualquer atividade física sem sintomas. Estes pacientes manifestam sinais de falência ventricular direita. Dispnéia e/ou fadiga podem estar presentes ao repouso e o desconforto aumenta em qualquer esforço.

A limitação da classe funcional como marcador isolado reside no fato de que ela depende do relato do paciente, que pode ser influenciado pela importância de determinadas atividades para si próprio, e da interpretação que o médico faz desse relato. Mas ainda assim, a variação da classe funcional foi representativa de melhora de sobrevida em vários estudos, devendo ser utilizada na avaliação de rotina dos pacientes. ${ }^{6}$

Dentre marcadores da capacidade funcional, o teste de caminhada de 6 minutos (TC6M) é um teste submáximo e constitui-se no principal objetivo primário dos principais ensaios clínicos realizados até o momento, tanto por sua simplicidade, quanto por sua correlação com parâmetros hemodinâmicos, com o teste de exercício máximo (teste cardiopulmonar de esforço), e por sua capacidade prognostica. ${ }^{6} 22,29$

O TC6M avalia a capacidade física e funcional, através das respostas globais e integradas dos sistemas envolvidos com o exercício (sistema pulmonar, cardiovascular e muscular), mas não fornece informações específicas da função de cada um desses sistemas. ${ }^{30}$ 
O TC6M deve refletir o nível de exercícios funcional para as atividades físicas diárias, já que a maior parte dessas atividades é realizada em um nível submáximo de exercício. É indicado para medir a resposta dos pacientes com doenças cardíacas ou pulmonares submetidos a intervenções medicamentosas e também como medida funcional. ${ }^{30}$

O TC6M é usado como marcador (end point) primário em pesquisas clínicas em HAP, sendo a única medida de capacidade de exercício aceita pelo FDA (Food and Drug Administration) para a liberação de novos medicamentos. O TC6M foi estudado principalmente em pacientes apresentando classe funcional (NYHA) III e IV, não tendo sido, ainda, validado como marcador em pacientes com doença menos avançada, com classe funcional I e II. ${ }^{31} 27$

\subsubsection{Avaliação da qualidade de vida}

A qualidade de vida é uma das medidas mais importantes de sucesso ou insucesso de um tratamento específico, principalmente sob a perspectiva do paciente. Atualmente, o objetivo do tratamento do paciente com HAP é aumentar a sobrevida e a sua qualidade de vida ${ }^{31}$.

Parece haver um consenso entre os estudiosos da área quanto a dois aspectos relevantes do conceito qualidade de vida: subjetividade e multidimensionalidade. A subjetividade é devido à percepção do indivíduo sobre o seu estado de saúde e sobre os aspectos não médicos do seu contexto de vida e a multidimensionalidade refere-se ao reconhecimento de que o construto é composto por diferentes dimensões. 
Duas tendências do termo na área da saúde são identificadas: qualidade de vida como um conceito mais genérico e qualidade de vida relacionada à saúde.

A qualidade de vida foi definida como "a percepção do indivíduo sobre a sua posição na vida, no contexto da cultura e dos sistemas de valores nos quais ele vive, e em relação aos seus objetivos, expectativas, padrões e preocupações". ${ }^{32}$

Em sua dimensão mais ampla, a qualidade de vida de um indivíduo é fortemente influenciada por fatores que não são relacionados diretamente pela sua saúde, incluindo sua situação econômica, moradia, emprego e suporte social. Uma denominação mais apropriada para designar a maneira como o cotidiano de um indivíduo é influenciado pelo seu estado de saúde seria "qualidade de vida relacionada à saúde" (QVRS). ${ }^{33} 34$

O termo QVRS, portanto, diz respeito à experiência subjetiva de como um impacto na saúde influencia a habilidade de um ser humano realizar e desfrutar as atividades de vida diária.

O termo "saúde" é formalmente definido pela Organização Mundial da Saúde como: "Um estado completo de bem-estar físico, mental e social, e não a mera ausência de doença ou enfermidade" 35

A avaliação da qualidade de vida relacionada à saúde é usada como forma a identificar a correlação entre a melhora da capacidade funcional e os demais aspectos relacionados à condição anterior ao tratamento, tais como distúrbios emocionais, limitação de atividades diárias e interação com a sociedade. ${ }^{36}$ 
Todo o questionário de QVRS deve ser definido em suas características antes que se possa avaliar com segurança as evidências coletadas por meio desse instrumento ${ }^{33,}{ }^{34}$. Três são os aspectos psicométricos considerados na caracterização dos instrumentos de avaliação de QVRS: reprodutibilidade ou confiabilidade, validade e responsividade.

A confiabilidade engloba a reprodutibilidade, possibilitando a repetição de resultados anteriormente encontrados quando da reaplicação do teste, com alta correlação inter e intra-observador. A confiabilidade também engloba a consistência interna que é a consistência de resposta dada a todos os itens de um determinado instrumento; quanto maior for a homogeneidade do conteúdo expresso através dos diferentes itens, menor será a variabilidade desta consistência e a quantidade de erros encontrados neste instrumento. Existem várias formas para avaliar a consistência interna de um instrumento, sendo a determinação do coeficiente alpha de Crombach, uma das mais utilizadas. ${ }^{37}$

A validade refere-se à capacidade que o instrumento tem ou não de medir aquilo a que se propõe e a responsividade é a capacidade do instrumento se ser sensível às alterações no quadro clínico ou bem-estar do paciente.

Vários questionários validados estão disponíveis para avaliação da QVRS, havendo instrumentos genéricos do estado de saúde e instrumentos específicos para determinadas doenças. ${ }^{33}$ 
Os instrumentos genéricos são aplicáveis a todas as populações, em indivíduos com ou sem doença. Fornecem a dimensão real para comparação entre diferentes grupos, como entre população saudável e doente ou mesmo diferentes faixas etárias, por exemplo. São exemplos de questionários genéricos: Nothing Health Profile (NHP) e Short Form 36 (SF36). 383940

Os instrumentos específicos são desenhados para avaliar indivíduos com determinada doença, ou sob um tratamento ou sintomas específicos. Eles oferecem uma avaliação mais detalhada e são mais sensíveis a alterações na QVRS decorrentes de uma intervenção. Exemplos de questionários específicos: Chronic Respiratory Questionnaire (CRQ) ${ }^{41}$, St. George Respiratory Questinnaire (SGRQ) ${ }^{42}$ e Airways Questionnaire20.(AQ-20) ${ }^{43}$.

Atualmente, questionários de qualidade de vida começam a ser avaliados como marcadores de gravidade e de resposta terapêutica na HAP. Entre os questionários genéricos de qualidade de vida existentes, o SF-36 é o mais utilizado. ${ }^{6}$

O SF-36 é um instrumento que nos últimos anos tem recebido grande atenção na literatura mundial devido ao seu desenho bem elaborado e às suas propriedades comprovadas de medida, reprodutibilidade, validade e responsividade. ${ }^{36,44}$

O SF-36 foi criado com a finalidade de ser um questionário genérico de avaliação de QVRS, de fácil administração e compreensão, porém sem ser tão extenso como os demais. O SF-36 vem sendo empregado por 
diversos pesquisadores em diferentes condições clínicas, tendo passado pelo processo de tradução e validação em diferentes línguas ${ }^{44-46}$. Uma vantagem adicional do uso do questionário SF-36 em nosso meio, é o fato de ele ser um dos poucos instrumentos disponíveis que sofreu um processo tecnicamente correto de tradução, adequação cultural e validação para a língua portuguesa falada no Brasil. ${ }^{47}$

O SF-36 é um questionário multidimensional, formado por 36 itens agrupados em oito escalas ou componentes (Anexo I): capacidade funcional, aspectos físicos, dor, estado geral de saúde, vitalidade, aspectos sociais, aspectos emocionais e saúde mental. ${ }^{47}$ Os 8 domínios do SF-36 podem ainda ser agrupados em dois grandes componentes: Componente Físico e Componente Mental. ${ }^{46}$

No momento do desenho desse estudo, nenhum instrumento específico par hipertensão pulmonar havia sido desenvolvido. Em 2006, foi publicado o primeiro estudo de validação do Cambridge Pulmonary Hypertension Outcome Review (CAMPHOR), realizado na Inglaterra, e em 2007 foi traduzido e validado para a população americana. A avaliação da QVRS pelo CAMPHOR consiste numa medida que avalie os sintomas, a capacidade funcional e um escore separado de qualidade de vida. ${ }^{48,49}$

Embora os diversos marcadores utilizados na avaliação de pacientes portadores de HAP sejam de fundamental importância para avaliação da gravidade da doença, do prognóstico e da resposta às diferentes intervenções terapêuticas existentes, à exceção da avaliação de qualidade de vida, nenhum deles reflete o impacto que a HAP tem no dia-a-dia dos 
pacientes, de forma objetiva. No momento em que esse estudo foi desenhado, não havia trabalhos com avaliação da qualidade de vida em HAP. Atualmente, os estudos publicados, alguns já com resultados parciais desse trabalho ${ }^{14,15}$, mostraram que 0 impacto da doença na qualidade de vida é tão importante quanto em outras doenças altamente limitantes como lesão medular, doenças intersticiais pulmonares ou ao câncer de próstata não-responsivo ao tratamento, por exemplo. ${ }^{50}$ Entretanto, ainda é necessário se estabelecer o real papel da avaliação da qualidade de vida como fator prognóstico e de resposta terapêutica em pacientes portadores de HAP. 


\section{Objetivos}


1 - Descrever e correlacionar a avaliação de qualidade de vida relacionada à saúde de pacientes com HAP com marcadores de gravidade funcionais e hemodinâmicos

2 - Avaliar o impacto da intervenção terapêutica específica na qualidade de vida de pacientes portadores de HAP

3 - Investigar o papel da avaliação de qualidade de vida como marcador prognóstico em pacientes com HAP. 
Métodos 


\subsection{POPULAÇÃO}

Foi feita a inclusão consecutiva de todos os pacientes com diagnóstico de HAP, do ambulatório do Grupo de Circulação Pulmonar da Disciplina de Pneumologia da Faculdade de Medicina da Universidade de São Paulo que foram submetidos ao cateterismo cardíaco direito no período de março de 2003 a setembro de 2007.

O projeto inicial previa a inclusão de 30 pacientes objetivando-se correlacionar a avaliação da qualidade de vida, com a distância caminhada no TC6M (com poder $=0,8, p=0,05$ e coeficiente de correlação mínimo esperado $=0,5$; com o advento de diferentes modalidades terapêuticas em nosso meio, optou-se pela expansão do estudo, através da inclusão consecutiva de todos os novos pacientes com HAP avaliados.

\subsection{CRITÉRIOS DIAGNÓSTICOS}

De acordo com o algoritmo proposto no Simpósio Internacional de Hipertensão Pulmonar, realizado em $2003^{5}$, foram definidos como critérios para o diagnóstico de HAPI os seguintes exames:

- radiografia de tórax - sem alteração no parênquima pulmonar;

- prova de função pulmonar - com capacidade vital forçada $>65 \%$ do valor previsto e relação entre volume expiratório forçado no primeiro segundo e a capacidade vital forçada > 70\%;

- angiotomografia de tórax sem presença de trombos (a angiografia convencional foi reservada aos casos em que houve dúvida na interpretação da angiotomografia); 
- ecocardiografia evidenciando aumento da pressão sistólica do ventrículo direito (maior ou igual a $40 \mathrm{mmHg}$ ), sem sinais de disfunção esquerda ou de cardiopatia congênita;

- ausência de sintomas compatíveis com apnéia obstrutiva do sono. $\mathrm{Na}$ presença de qualquer sinal clínico sugestivo, era solicitada uma polissonografia para confirmação diagnóstica;

- sorologias negativas para hepatites B e C e HIV;

- fatores reumatóide e anti-núcleo negativos. No caso de positividade de qualquer um desses marcadores, a investigação reumatológica era estendida a fim de afastar associação com doenças do tecido conectivo;

- exame protoparasitológico de fezes, com pesquisa negativa de ovos de Schistossoma mansoni. Realizou-se também exame ultrassonográfico de abdome superior para afastar a presença de achados característicos de esquistossomose, tais como aumento de lobo esquerdo do fígado ou presença de fibrose peri-portal e para afastar a presença de sinais compatíveis com hipertensão do sistema porta hepático, descartando-se o diagnóstico de hipertensão porto-pulmonar.

Todos os pacientes com suspeita de HAP realizaram ainda a avaliação hemodinâmica invasiva, considerando a presença de pressão média da artéria pulmonar $>25 \mathrm{mmHg}$ (em repouso) com pressão de oclusão da artéria pulmonar $<15 \mathrm{mmHg}$, como critério diagnóstico. 


\subsection{AVALIAÇÃO CLÍNICA E FUNCIONAL}

a) Avaliação hemodinâmica invasiva

A avaliação hemodinâmica é realizada de forma rotineira em todos os pacientes do ambulatório de circulação pulmonar da Disciplina de Pneumologia da Faculdade de Medicina da Universidade de São Paulo, para diagnóstico de hipertensão arterial pulmonar.

A avaliação hemodinâmica invasiva é realizada nos pacientes respirando em ar ambiente, na posição supina. Através de um cateter de artéria pulmonar é medido o débito cardíaco (DC) através da técnica de termodiluição.

Para este estudo, os seguintes dados hemodinâmicos foram coletados do cateterismo realizado durante a avaliação diagnóstica:

- pressão de átrio direito

- pressão média da artéria pulmonar

- pressão de oclusão da artéria pulmonar

- débito cardíaco

- resistência vascular pulmonar

b) Classe funcional

A estratificação dos pacientes foi realizada através da Classificação Funcional da New York Heart Association (NYHA), modificada para hipertensão pulmonar, na consulta de inclusão do paciente no estudo. ${ }^{51}$ 
c) Teste de caminhada de 6 minutos

O TC6M foi realizado em um período de no máximo 7 dias em relação à avaliação hemodinâmica, de acordo com as diretrizes estabelecidas pela American Thoracic Society (ATS) ${ }^{30}$. Foi realizado em um corredor de 30 metros, com monitorização contínua da freqüência cardíaca (FC) e saturação de oxigênio $\left(\mathrm{SatO}_{2}\right)$ durante o percurso e medida a dispnéia inicial e final através da Escala de Dispnéia de Borg ${ }^{52,53}$. A distância percorrida ao final dos 6 minutos foi anotada, independente de qualquer parada durante o teste. Nos pacientes submetidos a tratamento específico, o TC6M foi realizado novamente após 16 semanas do início do tratamento.

d) Avaliação da Qualidade de Vida

Para avaliação da qualidade de vida relacionada à saúde foi utilizado o questionário de qualidade de vida Medical Outcomes Study 36-item ShortForm Health (SF-36) (Anexo I). O SF-36 é formado por 36 itens agrupados em oito domínios ou componentes: capacidade funcional, aspectos físicos, dor, estado geral de saúde, vitalidade, aspectos sociais, aspectos emocionais e saúde mental. Cada domínio apresenta uma escala com valor de 0 a 100, onde zero corresponde à pior condição de qualidade de vida e 100 à melhor.

Embora o questionário tenha sido desenvolvido para auto-avaliação, considerando as características sócio-culturais da população de nosso hospital, optou-se pela administração do mesmo por uma única pessoa com experiência em avaliação em qualidade de vida. Os pacientes foram 
instruídos pelo entrevistador a responder as 11 questões relacionadas aos 8 diferentes domínios de qualidade de vida. Os pacientes foram questionados a pontuar suas respostas de acordo com uma escala definida em cada questão. Os escores e a interpretação dos resultados eram estritamente aderentes às diretrizes da Pontuação do Questionário SF-36 (Apêndice 1.1) e Cálculo dos Escores para o Questionário SF-36 (Apêndice 1.2).

Após a inclusão do paciente no estudo, o questionário SF-36 foi aplicado no mesmo dia que foi realizado o teste de caminhada de 6 minutos. E a mesma avaliação foi realizada após 16 semanas de qualquer tratamento específico iniciado.

\subsection{ANÁLISE ESTATÍSTICA}

Os dados contínuos estão apresentados sob a forma de média (desvio padrão) enquanto que os categóricos sob a forma de proporção, exceto quando explicitado de outra forma. O coeficiente alfa de Cronbach foi calculado para todos os escores do SF-36 para avaliar sua confiabilidade e consistência interna, sendo que um valor de $\alpha>0,7$ foi considerado como satisfatório. ${ }^{54} 55$

Foi utilizado o método de Pearson para avaliar a correlação entre os escores de qualidade de vida e os demais marcadores de gravidade estudados. A comparação dos dados obtidos na avaliação basal e após 16 semanas de tratamento específico foi realizada através do teste t pareado. No subgrupo de pacientes tratados, a sobrevida foi descrita através de curvas de Kaplan-Meyer. Após a categorização das curvas pela mediana 
dos escores de qualidade de vida, a comparação entre as curvas de sobrevida foi realizada através do teste de log-rank. Os resultados foram considerados significativos quando o valor de p era menor que 0,05. 


\section{Resultados}


As características clínicas dos 126 pacientes incluídos no estudo estão apresentadas na tabela 1 . O grau de acometimento hemodinâmico está de acordo com os dados previamente publicados em HAP.

Tabela 1. Dados clínicos e hemodinâmicos basais. $(n=126)$

\begin{tabular}{lc}
\hline Idade (anos) & $46(13)$ \\
\hline Sexo (f/m) & $96 / 30$ \\
\hline Diagnóstico n(\%) & $67(53)$ \\
$\quad$ Idiopática & $29(23)$ \\
Esquistossomose & $15(12)$ \\
Colagenose & $6(5)$ \\
ClA/ Cardiopatia & $9(7)$ \\
Outros & $32(25)$ \\
\hline Classe Funcional n(\%) $\quad$ II & $60(48)$ \\
$\quad$ IV & $34(27)$ \\
\hline Distância TC6M (m) & $412(125)$ \\
\hline Pressão média da artéria pulmonar (mmHg) & $61(19)$ \\
\hline Pressão de oclusão da artéria pulmonar (mmHg) & $10(2)$ \\
\hline Débito Cardíaco (L/min) & $4,17(1)$ \\
\hline Resistência Vascular Pulmonar (dina.cm $\left.{ }^{-5} . \mathrm{s} . \mathrm{m}^{-2}\right)$ & $13,89(9)$
\end{tabular}

A confiabilidade da consistência interna do questionário SF-36 se mostrou adequada com $\alpha$ de Cronbach $=0,852$.

Os escores de qualidade de vida dos oito domínios e dos componentes físico e mental (tabela 2 e, graficamente, na figura 2) apresentaram valores menores do que da população normal dos EUA, Canadá e do Reino Unido de acordo com a literatura. Como está demonstrado no Anexos 1 e 2. ${ }^{50}$ 
Tabela 2. Escores da Qualidade de Vida. $(n=126)$

\begin{tabular}{lcc}
\hline Capacidade Funcional & $33(22)$ & $0-95$ \\
\hline Aspectos Físicos & $28(36)$ & $0-100$ \\
\hline & $59(27)$ & $0-100$ \\
\hline Dor & $46(20)$ & $0-97$ \\
\hline Estado Geral da Saúde & & $0-100$ \\
\hline & $43(24)$ & $0-100$ \\
\hline Vitalidade & $55(28)$ & $0-100$ \\
\hline Aspectos Sociais & $52(39)$ & $0-100$ \\
\hline Aspectos Emocionais & $57(24)$ & \\
\hline Saúde Mental & $9-54$ \\
\hline Componente Físico & $33(9)$ & $16-72$ \\
\hline Componente Mental & $44(12)$ & \\
\hline
\end{tabular}

Resultados apresentados em média (desvio padrão) e min - max. 

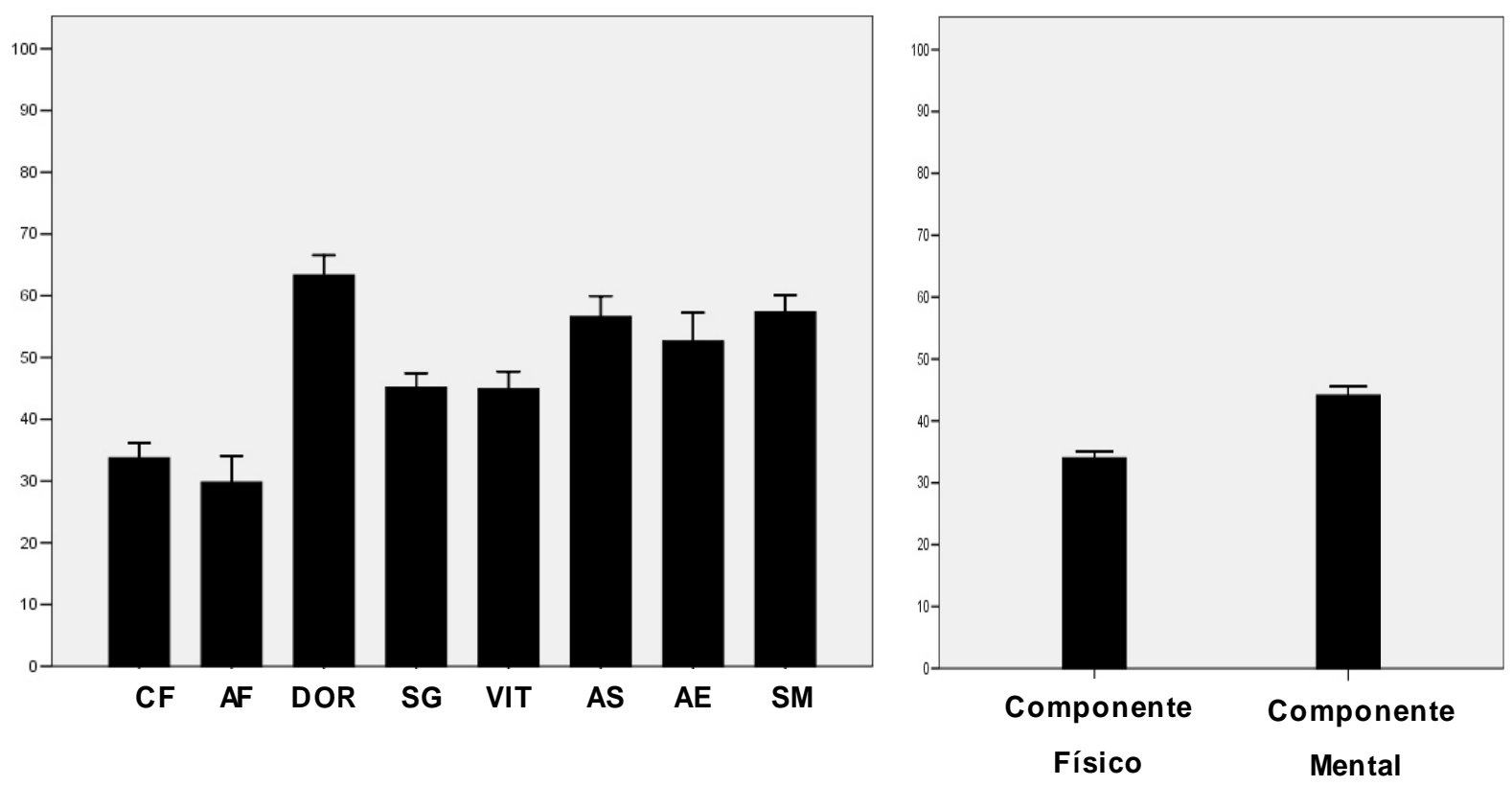

Figura 2: Escores dos domínios de qualidade de vida representados pelos valores médios (erro padrão da média).

CF: Capacidade Funcional

AF: Aspectos Físicos

SG: Saúde Geral

DOR: Dor

VIT: Vitalidade

AS: Aspectos Sociais

AE: Aspectos Emocionais

SM: Saúde Mental 
A análise entre a avaliação de qualidade de vida e as medidas hemodinâmicas não evidenciou qualquer correlação significante, a não ser pela tênue correlação entre o domínio de capacidade funcional com o débito cardíaco ( $p=0,002, r=0,28)$ (Figura 3) e com a resistência vascular pulmonar $(p=0,42, r=-0,19)$.

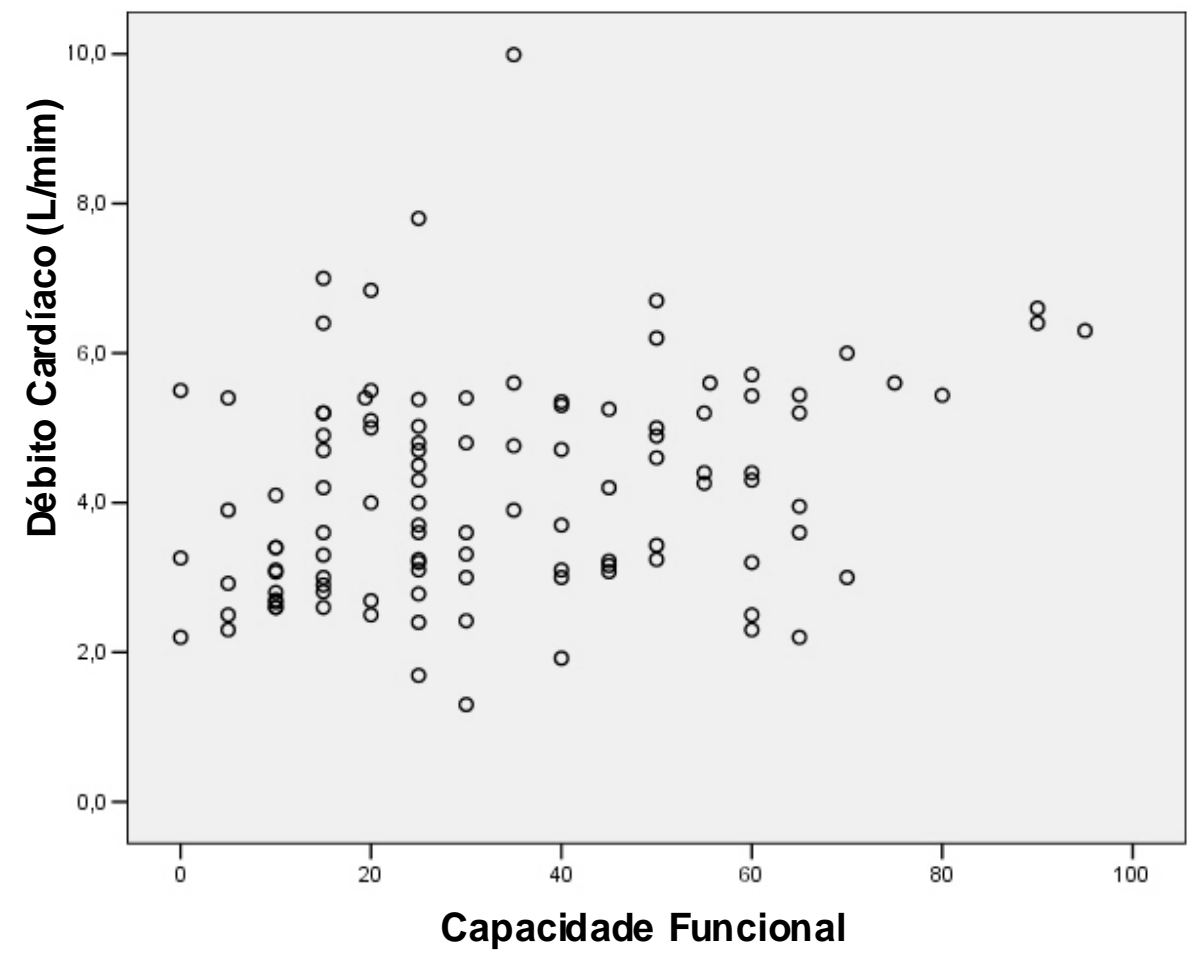

Figura 3: Correlação entre débito cardíaco e a capacidade funcional da qualidade de vida. $(p=0,002, r=0,28)$. 
Todos os domínios da qualidade de vida apresentaram correlação significativa com a Classe Funcional (NYHA), conforme descrito na tabela 3. Os componentes físico e mental podem ser visualizados nas figuras $4 \mathrm{~A}$ e 4B.

Tabela 3. Correlação da Classe Funcional com a qualidade de vida.

\begin{tabular}{lcc}
\hline & $\mathrm{R}$ & $\mathrm{p}$ \\
\hline Capacidade Funcional & $-0,52$ & $<0,001$ \\
\hline Aspectos Físicos & $-0,47$ & $<0,001$ \\
\hline Dor & $-0,32$ & $<0,001$ \\
\hline Estado Geral da Saúde & $-0,33$ & $<0,001$ \\
\hline Vitalidade & $-0,41$ & $<0,001$ \\
\hline Aspectos Sociais & $-0,42$ & $<0,001$ \\
\hline Aspectos Emocionais & $-0,27$ & 0,002 \\
\hline Saúde Mental & $-0,19$ & 0,03 \\
\hline Componente Físico & $-0,52$ & $<0,001$ \\
\hline Componente Mental & $-0,24$ & 0,006 \\
\hline
\end{tabular}



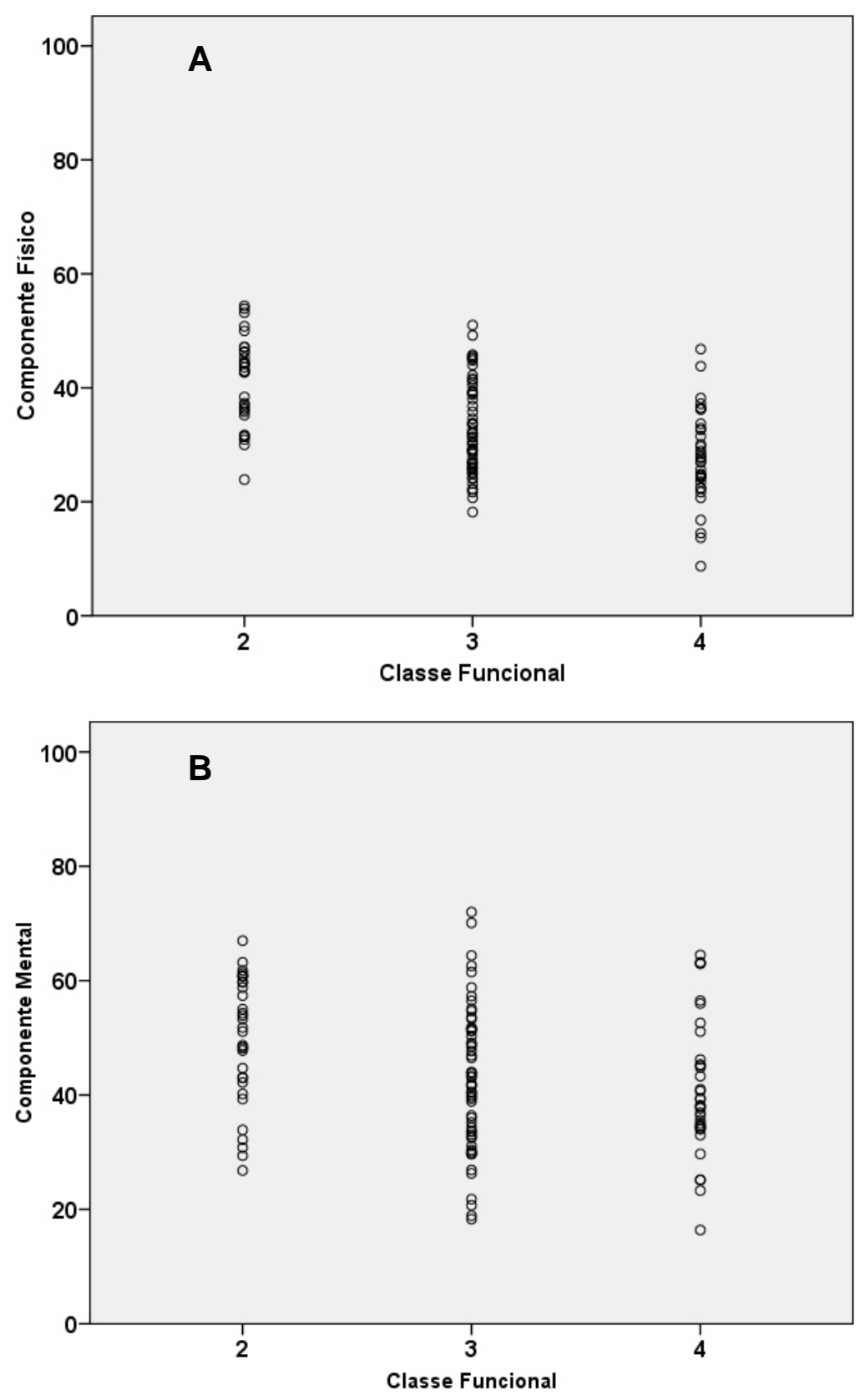

Figura 4: A: Correlação entre Classe Funcional e o Componente Físico ( $p<$ 0,001, r= - 0,52). B: Correlação entre Classe Funcional e o Componente Mental $(p=0,006, r=-0,24)$ 
O TC6M também apresentou correlação com todos os escores de qualidade de vida, conforme demonstrado na tabela 4. Os domínios físicos apresentam melhor correlação do que os domínios emocionais e mentais. A correlação do TC6M com o componente físico pode ser visualizada na Figura 5.

Tabela 4. Correlação do TC6M com a qualidade de vida.

\begin{tabular}{|c|c|c|}
\hline & $\mathrm{R}$ & $p$ \\
\hline Capacidade Funcional & 0,58 & $<0,001$ \\
\hline Aspectos Físicos & 0,33 & $<0,001$ \\
\hline Dor & 0,35 & $<0,001$ \\
\hline Estado Geral da Saúde & 0,24 & 0,005 \\
\hline Vitalidade & 0,37 & $<0,001$ \\
\hline Aspectos Sociais & 0,43 & $<0,001$ \\
\hline Aspectos Emocionais & 0,23 & 0,009 \\
\hline Saúde Mental & 0,25 & 0,005 \\
\hline Componente Físico & 0,48 & $<0,001$ \\
\hline Componente Mental & 0,26 & 0,003 \\
\hline
\end{tabular}




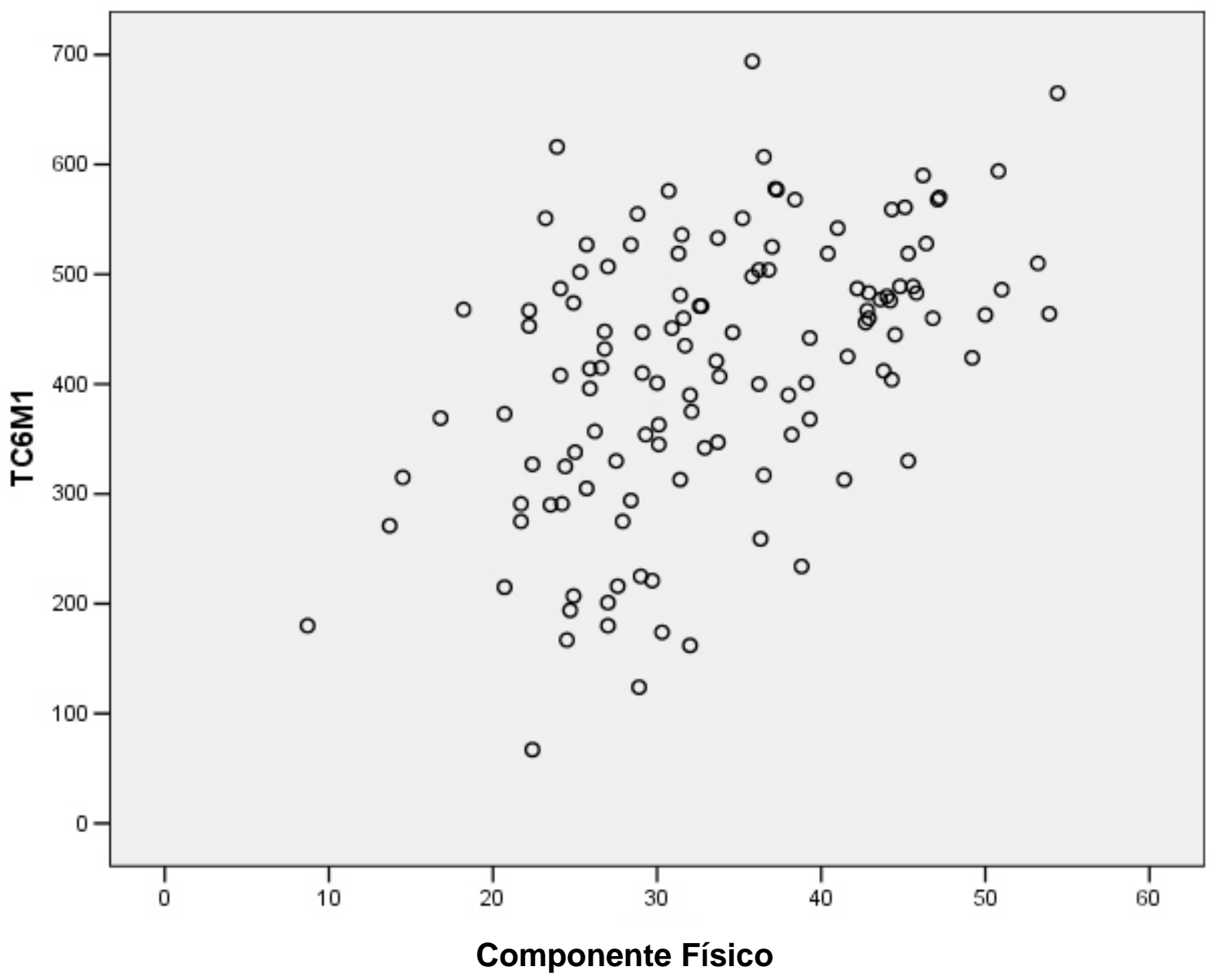

Figura 5. Correlação entre componente físico e distância do teste de caminhada de 6 minutos. $(r<0,001, r=0,483)$. 
Do grupo total de pacientes estudados, cinqüenta e quatro foram submetidos a tratamento específico para HAP. As características clínicas desse subgrupo estão descritas na tabela 4.

Tabela 5. Dados clínicos e hemodinâmicos do subgrupo tratado $(n=54)$.

\begin{tabular}{|c|c|}
\hline Idade (anos) & $44(12)$ \\
\hline $\operatorname{Sexo}(f / m)$ & $45 / 9$ \\
\hline \multicolumn{2}{|l|}{ Diagnóstico n(\%) } \\
\hline $\begin{array}{l}\text { Idiopática } \\
\text { Esquistossomose } \\
\text { Colagenose } \\
\text { CIA/ Cardiopatia }\end{array}$ & $\begin{array}{l}37(68) \\
7(13) \\
8(15) \\
2(4) \\
\end{array}$ \\
\hline $\begin{array}{cc}\text { Classe Funcional } & \text { n (\%) } \\
& \text { II } \\
\text { III } \\
\text { IV } \\
\end{array}$ & $\begin{array}{l}14(26) \\
28(52) \\
12(22) \\
\end{array}$ \\
\hline Distância TC6M (m) & $418(121)$ \\
\hline Pressão média da artéria pulmonar $(\mathrm{mmHg})$ & $64(17)$ \\
\hline Pressão de oclusão da artéria pulmonar $(\mathrm{mmHg})$ & $10(2)$ \\
\hline Débito Cardíaco (L/min) & $4(1)$ \\
\hline Resistência Vascular Pulmonar (dina.cm ${ }^{-5} . \mathrm{s} \cdot \mathrm{m}^{-2}$ ) & $15(8)$ \\
\hline \multicolumn{2}{|l|}{ Tratamento $\mathrm{n}(\%)$} \\
\hline $\begin{array}{l}\text { Bosentan } \\
\text { Sitaxsentan } \\
\text { Ambrisentan } \\
\text { Sildenafil }\end{array}$ & $\begin{array}{c}16(29) \\
27(50) \\
3(5) \\
8(15)\end{array}$ \\
\hline
\end{tabular}


Nesses 54 pacientes, a avaliação da qualidade de vida foi repetida após 16 semanas de tratamento, evidenciando melhora significativa em todos os escores, exceto no domínio da dor (tabela 5). O mesmo dado pode ser analisado de forma gráfica na figura 6.

Tabela 6. Escores da Qualidade de Vida. $(n=54)$

\begin{tabular}{lccc}
\hline & Pré & pós & P \\
\hline Capacidade Funcional & $33(21)$ & $41(20)$ & $\mathrm{p}<0,001$ \\
\hline Aspectos Físicos & $29(37)$ & $50(39)$ & $\mathrm{p}=0,001$ \\
\hline Dor & $63(28)$ & $66(25)$ & $\mathrm{p}=0,174$ \\
\hline Estado Geral da Saúde & $45(20)$ & $53(18)$ & $\mathrm{p}=0,003$ \\
\hline Vitalidade & $44(24)$ & $58(21)$ & $\mathrm{p}<0,001$ \\
\hline Aspectos Sociais & $56(29)$ & $68(26)$ & $\mathrm{p}=0,004$ \\
\hline Aspectos Emocionais & $52(40)$ & $67(37)$ & $\mathrm{p}=0,007$ \\
\hline Saúde Mental & $57(24)$ & $67(19)$ & $\mathrm{p}=0,002$ \\
\hline Componente Físico & $34(9)$ & $37(8)$ & $\mathrm{p}=0,005$ \\
\hline Componente Mental & $44(12)$ & $50(10)$ & $\mathrm{p}<0,001$ \\
\hline Resultados apresentados & & &
\end{tabular}

Resultados apresentados em média (DP).

O teste de caminhada de 6 minutos apresentou aumento significativo na distância percorrida de 414 (124) para 440 (113) metros no pós tratamento $(p=0,001)$. 

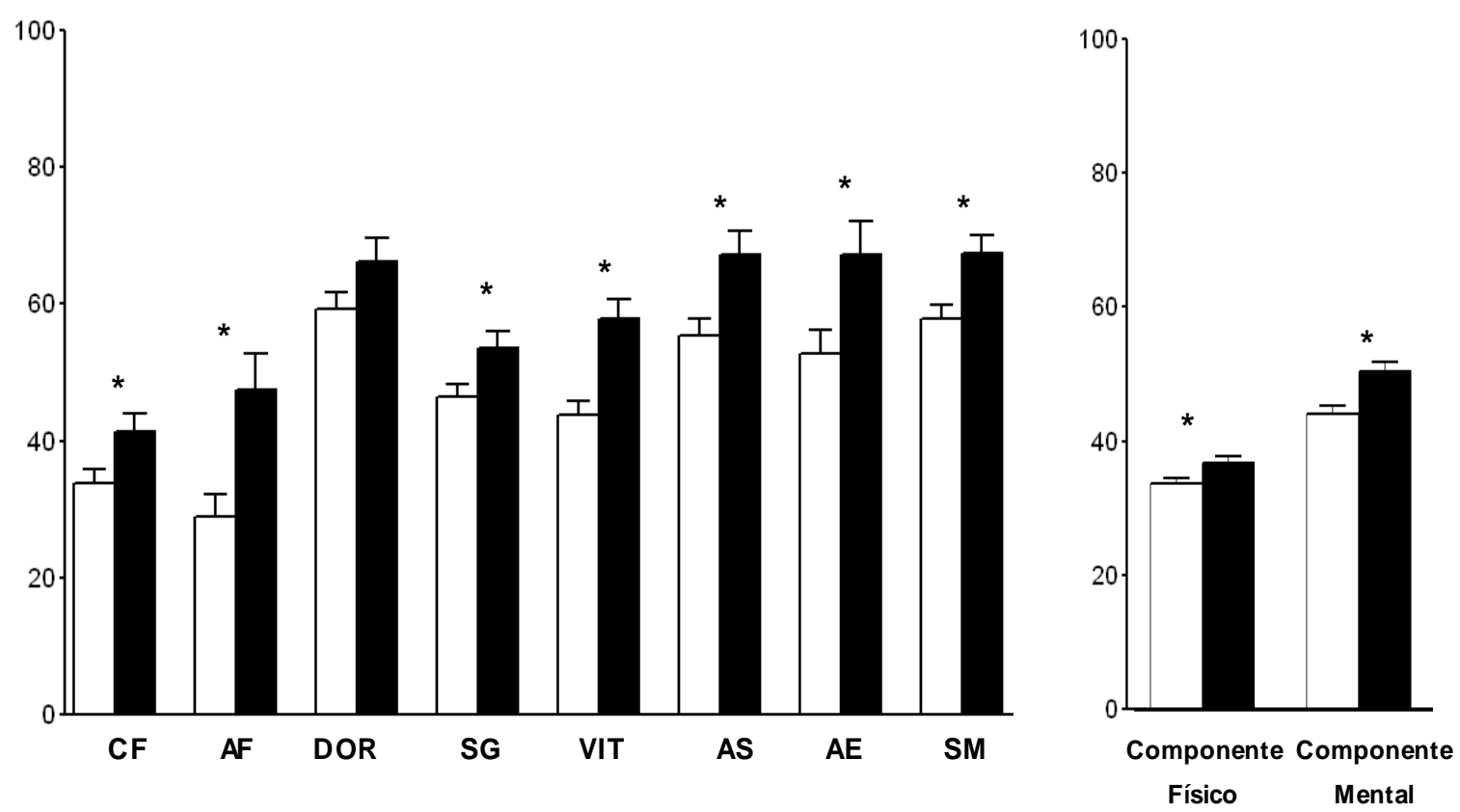

Figura 6. Variação nos escores do questionário de qualidade de vida após 16 semanas de tratamento. $\left({ }^{*} p<0,05\right)$

CF: Capacidade Funcional

AF: Aspectos Físicos

SG: Saúde Geral

DOR: Dor

VIT: Vitalidade

AS: Aspectos Sociais

AE: Aspectos Emocionais

SM: Saúde Mental 
$\mathrm{Na}$ avaliação dos escores de qualidade de vida como marcador prognóstico, foram incluídos os 54 pacientes que receberam tratamento específico para HAP. Os dados mostraram que os pacientes que obtiveram escore >32 no Componente Físico, na avaliação basal, apresentaram melhor sobrevida. (Figura 7). Os demais escores e mesmo o componente mental não evidenciaram relação com o prognóstico.

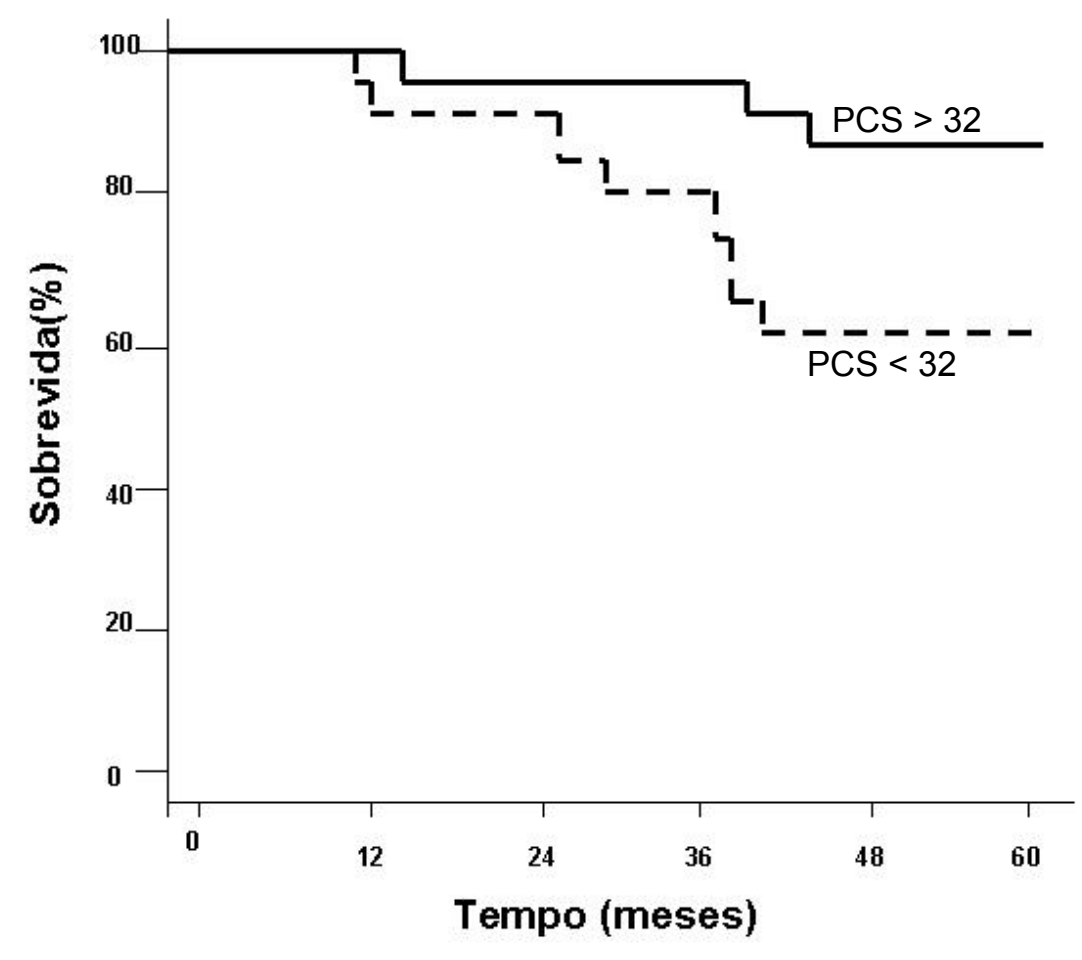

Figura 7. Curva de sobrevida em função do tempo de acordo com o escore do componente físico. $(p=0,04)$ PCS ( Physical Component Sumary): Componente Físico 
A análise da variação do componente físico, estratificado de acordo com a mediana, após 16 semanas de tratamento, não apresentou correlação com a sobrevida (Figura 8).

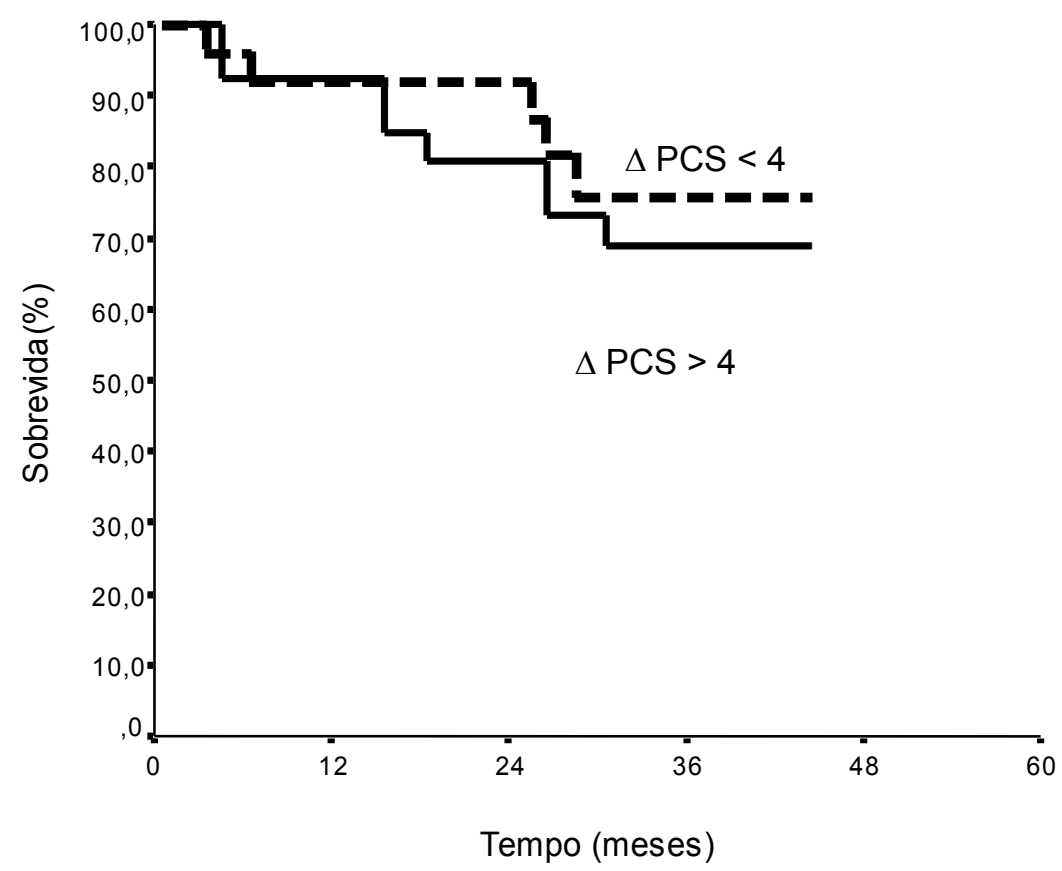

Figura 8. Curva de sobrevida em função do tempo de acordo com a variação do escore de Componente Físico $(p=0,58)$.

PCS ( Physical Component Sumary): Componente Físico 
Entretanto, analisando a melhora do escore do Componente Físico após 16 semanas de tratamento, os pacientes que atingiram o escore do Componente Físico > 38 apresentaram melhor prognóstico que os pacientes que não atingiram esse valor (Figura 9).

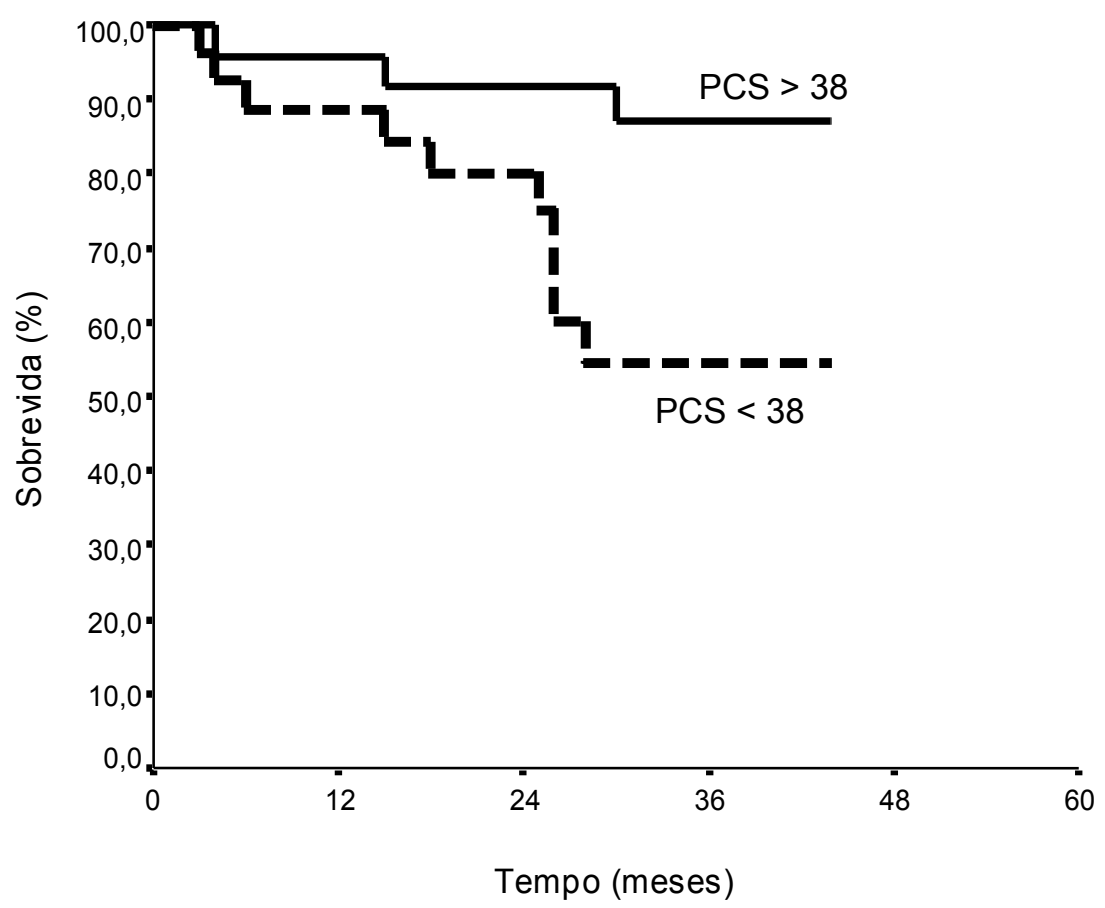

Figura 9. Curva de sobrevida em função do tempo de acordo com o escore Componente Físico após 16 semanas de tratamento $(p=0,016)$. PCS ( Physical Component Sumary): Componente Físico 
Discussão 
Os resultados desse estudo demonstraram que a avaliação da qualidade de vida relacionada à saúde realizada através do questionário SF36 apresenta correlação significativa com marcadores funcionais e hemodinâmicos em pacientes com HAP e é capaz de refletir o impacto do tratamento específico na qualidade de vida desse pacientes. Além disso, o componente físico se correlacionou com a sobrevida, mostrando-se como marcador de gravidade da doença.

A HAP é uma situação clínica de alta gravidade, caracterizada pela presença de vasoconstrição pulmonar, trombose in situ e remodelamento vascular levando a progressiva insuficiência ventricular direita e ao óbito. Essa progressão é particularmente importante se considerarmos a alta mortalidade associada a HAP; a sobrevida média era de 2,8 anos após diagnóstico, antes do surgimento e uso rotineiro das novas drogas disponíveis.

Uma característica importante de nossa amostra reside no fato do critério diagnóstico ter sido baseado nas medidas invasivas, garantindo assim a acurácia diagnóstica. Os dados demográficos encontrados em nosso estudo são comparáveis a outras populações de pacientes com HAP previamente descritas. 505657

Entretanto, uma característica peculiar da população brasileira merece ser ressaltada. Dentre os diferentes diagnósticos, mais da metade dos pacientes eram portadores de HAPI e 12\% apresentavam HAP associada à doença do tecido conjuntivo; todavia, 23\% dos pacientes tinham esquistossomose como fator etiológico associado, o que é esperado em 
função da prevalência desta doença em país, e em concordância com os achados do estudo de Lapa e col. que caracterizou a população com HAP de dois centros de referência de São Paulo. ${ }^{9} \mathrm{~A}$ maioria dos pacientes apresentava classe funcional III e IV, à inclusão, o que reflete indivíduos com maior gravidade da doença. ${ }^{57,58}$ A limitação do uso da Classe Funcional em relação à avaliação da qualidade de vida deve-se ao fato de ser resultado da interpretação do médico sobre o relato subjetivo do paciente que pode ser influenciado pela importância de determinadas atividades para si próprio. ${ }^{27}$ Mesmo assim, a proporção de pacientes em classes funcionais mais avançadas reflete uma característica ainda importante da HAP que é o diagnóstico tardio, em relação ao início dos sintomas.

Apesar da maior parte dos pacientes se encontrar em classes funcionais avançadas, a distância média caminhada durante o TC6M foi de 412 metros, o que caracteriza melhor prognóstico, segundo dados descritos anteriormente por Miyamoto e col. ${ }^{22}$

Atualmente, a avaliação da qualidade de vida é uma das medidas mais importantes de sucesso ou falência de uma determinada terapia porque avalia o impacto dos danos e incapacidades na vida dos pacientes. ${ }^{31,59}$ Entre os instrumentos utilizados para avaliação da qualidade de vida relacionada à saúde, genéricos ou específicos, optamos por utilizar um instrumento genérico, pois no momento da concepção desse estudo não havia relatos na literatura de um questionário específico validado para HAP. Em 2006, no Reino Unido, foi desenvolvido e validado um questionário de avaliação de qualidade de vida específico para pacientes com hipertensão 
pulmonar, The Cambrige Pulmonary Hypertension Outcome Review (CAMPHOR). ${ }^{49} \mathrm{O}$ CAMPHOR tem mostrado uma medida aceitável de qualidade de vida e capaz de avaliar o impacto de intervenções nos sintomas e capacidade funcional. ${ }^{49} 48$ Entretanto, o CAMPHOR ainda necessita ser traduzido e validado para a língua portuguesa, o que seria interessante, para comparar com os resultados obtidos no nosso estudo e avaliar sua capacidade prognóstica.

O questionário escolhido foi o SF-36 (Medical Outcomes Study 36item Short Form) porque está devidamente traduzido e validado na língua portuguesa. O SF-36 é um questionário de fácil administração e compreensão e tem sido amplamente utilizado em diversos países, populações e situações clínicas, abrindo a possibilidade para comparar nossos resultados com outras populações.

Não existe no Brasil uma avaliação de qualidade de vida relacionada à saúde que determine a normalidade da população geral. Apesar deste não ser o intuito desse trabalho, para efeitos de comparação, apresentamos os resultados dos escores dos oito domínios do SF-36 descritos em diferentes populações normais dos EUA, Canadá, Reino Unido, ${ }^{60}$ além uma amostra da população de Montes Claros retirados de uma tese de doutorado da UNIFESP ${ }^{61}$ e de um pequeno grupo de 40 indivíduos sem comorbidades avaliados por nós. Nossos dados de normalidade estão de acordo com os dados americano e canadense, apesar de não podermos tirar conclusões estatísticas devido ao tamanho de nossa amostra. Os componentes físico e mental do nosso grupo de normalidade apresentaram valores 51 e 50, 
respectivamente, portanto de acordo com os critérios de normalização dos dados, originalmente realizados para a população norte-americana.

Comparando os dados do grupo de pacientes com HAP de nosso estudo com as populações normais, todos os escores dos 8 domínios e 2 componentes estão diminuídos, como ilustrado no anexo 3. O escore que apresentou menor impacto foi o de dor, o que pode ser explicado pelo fato de dor não ser um dos sintomas característicos da HAP.

Para validar o SF-36 na HAP, avaliamos sua correlação com marcadores hemodinâmicos e funcionais pré-estabelecidos. Em relação às medidas hemodinâmicas encontramos apenas uma tênue correlação do domínio da capacidade funcional com débito cardíaco e com a resistência vascular pulmonar, não havendo correlação significante dos demais escores com as medidas hemodinâmicas, em conformidade com o que foi previamente descrito por Taichman e col. ${ }^{50}$ Os marcadores funcionais apresentaram forte correlação com os domínios da avaliação de qualidade de vida. Todos os 8 domínios apresentaram correlação significativa com a Classe Funcional (NYHA), sendo que o domínio relacionado à capacidade funcional e o relacionado aos aspectos físicos, e conseqüentemente o componente físico, tiveram melhor correlação. A vitalidade e aspectos sociais também apresentam boa correlação com a Classe Funcional, o que poderia ser explicado pelo fato de os pacientes que apresentam piores condições físicas ficam mais limitados, diminuindo assim sua atividade social. 
Outro marcador funcional na HAP com papel bem estabelecido como marcador de gravidade e prognóstico é o TC6M, que apresentou forte correlação com todos os escores de qualidade de vida. E da mesma forma que a classe funcional, também apresentou melhor correlação com os domínios relacionados à parte física, principalmente a capacidade funcional e componente físico. Esses achados sugerem que o estado da capacidade funcional tem grande impacto na qualidade de vida do indivíduo, maior do que pode ser avaliado pelas medidas hemodinâmicas e confirmam a importância da qualidade de vida relacionada à saúde como marcador nas pesquisas clínicas, pois a melhora no escore de qualidade de vida reflete a sensação de bem-estar do paciente.

Do grupo total de pacientes estudados, 54 foram submetidos a tratamento específico para HAP e a qualidade de vida relacionada à saúde e TC6M avaliados novamente. O subgrupo se manteve com as mesmas características demográficas e de gravidade em relação ao grupo total.

O TC6M e a avaliação da qualidade de vida apresentaram melhora significativa. O único domínio que teve uma melhora menos evidente foi o domínio da dor, o qual já na avaliação basal era o menos acometido nos pacientes com HAP. Os componentes físico e mental, apesar de apresentarem um aumento pequeno em valor absoluto, apresentaram significância estatística, pois seu valor é normatizado para 50; desta forma, a variação esperada sempre é menor que a variação dos domínios isoladamente. Esses dados confirmam as análises preliminares publicadas por nosso grupo, ao longo do estudo. ${ }^{14,15}$ 
Para avaliar o papel prognóstico da qualidade de vida, decidimos categorizar os pacientes, que foram submetidos a tratamento, de acordo com a mediana de cada domínio e componente. O componente físico foi o único escore que apresentou correlação significativa com a sobrevida através da curva de Kaplan-Meyer, onde os pacientes que obtiveram escore > 32 evidenciaram melhor prognóstico. Não é de nosso conhecimento qualquer estudo que tenha evidenciado o papel da avaliação da qualidade de vida na avaliação prognóstica de pacientes com HAP.

Após 16 semanas de tratamento, a melhora na qualidade de vida foi significante, mas analisando a sobrevida em função da mediana da variação absoluta do componente físico, não se evidenciou significância estatística. Todavia, quando a mediana do componente físico encontrado após 16 semanas de tratamento foi utilizada, houve associação com melhor prognóstico.

Esses resultados sugerem que a avaliação da qualidade de vida através do Componente Físico se comporta como marcador de resposta similar ao TC6M. Demonstrou-se anteriormente que a variação absoluta do TC6M não apresenta correlação com a sobrevida; entretanto, quando um determinado valor era atingido após o tratamento, independentemente da variação absoluta, havia melhor prognóstico. ${ }^{28}$ Esse foi o comportamento do componente físico, em nosso estudo. Os pacientes que atingiram o valor de 38 no componente físico, após 16 semanas de tratamento específico, tiveram melhor prognóstico, independente da variação absoluta do componente físico em função do tratamento. 
Por ser esta a primeira descrição da capacidade prognóstica da avaliação da qualidade de vida em pacientes com HAP, os valores de corte apresentados necessitam de validação em outras amostras para que possam ser extrapolados para a população de pacientes com HAP. Independente dessa limitação de nosso estudo, nossos resultados já evidenciam que a avaliação da qualidade de vida se mostra como marcador de gravidade da doença.

Concluímos, portanto, que a avaliação de qualidade de vida é importante fonte de informações clínicas e prognósticas adicionais em pacientes com HAP, avaliando de forma objetiva a experiência subjetiva de como a HAP ou a sua terapêutica tem impacto na habilidade do paciente em realizar e desfrutar as atividades de vida diária. 
Conclusão 
A avaliação da qualidade de vida dos pacientes com HAP, através do questionário SF-36:

- Correlaciona-se com outros marcadores funcionais e hemodinâmicos previamente reconhecidos.

- Reflete o impacto de intervenção terapêutica específica.

- Tem papel prognóstico, mais especificamente através da avaliação de seu componente físico. 
Anexos 


\section{Anexo1: Tabela da Qualidade de Vida}

HCFMUSP $\begin{gathered}\text { Montes } \\ \text { Claros } \\ \text { (UNIFESP) }\end{gathered}$ Canadá $^{60}$ EUA $^{60} \begin{gathered}\text { Reino } \\ \text { Unido }^{60}\end{gathered}$

\begin{tabular}{llllll}
\hline Capacidade Funcional & 84 & 83 & 88 & 85 & 89 \\
\hline
\end{tabular}

\begin{tabular}{llllll}
\hline Aspectos Físicos & 89 & 87 & 74 & 82 & 89 \\
\hline & & & & & \\
\hline Dor & 69 & 73 & 86 & 75 & 78 \\
\hline
\end{tabular}

\begin{tabular}{llllll}
\hline Estado Geral da Saúde & 80 & 75 & 77 & 72 & 71 \\
\hline
\end{tabular}

\begin{tabular}{|c|c|c|c|c|c|}
\hline Vitalidade & 66 & 70 & 65 & 61 & 59 \\
\hline Aspectos Sociais & 84 & 84 & 86 & 84 & 83 \\
\hline
\end{tabular}

\begin{tabular}{llllll}
\hline Aspectos Emocionais & 78 & 86 & 75 & 81 & 86 \\
\hline
\end{tabular}

\begin{tabular}{lccccc}
\hline Saúde Mental & 76 & 73 & 76 & 74 & 72 \\
\hline Componente Físico & 51 & & 51 & & \\
\hline Componente Mental & 50 & & & & \\
\hline
\end{tabular}


ANEXO 2: Qualidade de vida da população normal

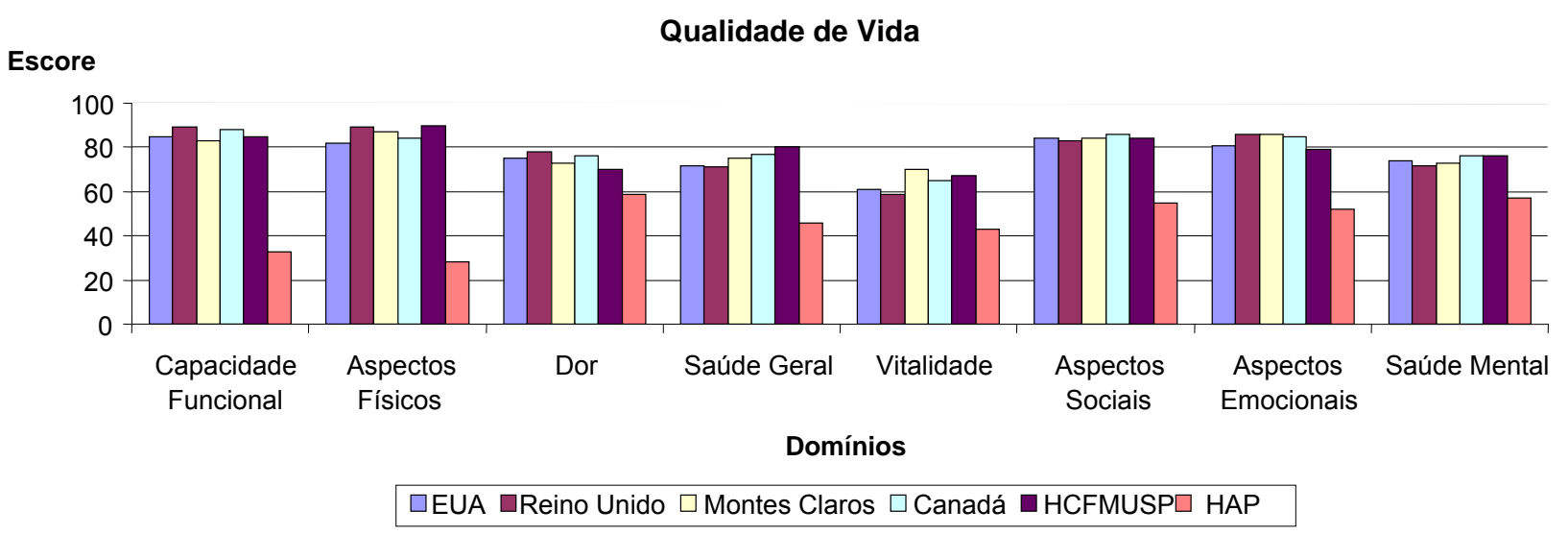

\section{ANEXO 3: Qualidade de vida relacionada à saúde}

\section{Qualidade de Vida}

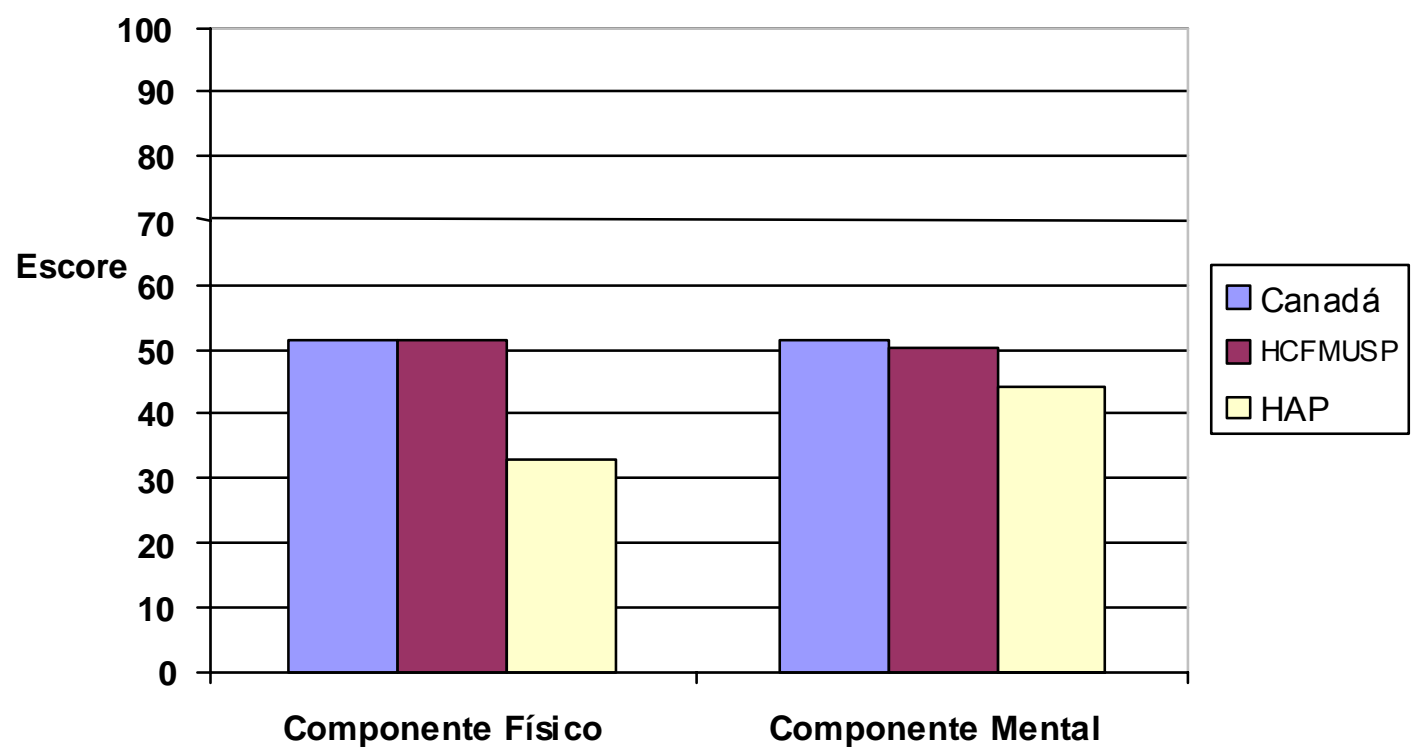

Componentes 


\section{Referências}


1. Rich, S, Dantzker, D.R., Ayres, S.M., Bergofsky, E.H., Brundage, B.H., Detre, K.M., Fishman, A.P., Goldring, R.M., Groves, B.M., Koerner, S.K., and et al, Primary pulmonary hypertension. A national prospective study. Ann Intern Med, 1987: p. 216-23.

2. Rubin, LJ, Diagnosis and management of pulmonary arterial hypertension: ACCP evidence-based clinical practice guidelines. Chest, 2004. 126: p. 4S-6S.

3. Simonneau, G, Galie, N, Rubin, LJ, Langleben, D, Seeger, W, Domenighetti, G, Gibbs, S, Lebrec, D, Speich, R, Beghetti, M, Rich, S, and Fishman, A, Clinical classification of pulmonary hypertension. J Am Coll Cardiol, 2004. 43: p. 5S-12S.

4. Rubin, LJ, Primary pulmonary hypertension. N Engl J Med, 1997. 336: p. 111-7.

5. Barst, RJ, McGoon, M, Torbicki, A, Sitbon, O, Krowka, MJ, Olschewski, H, and Gaine, S, Diagnosis and differential assessment of pulmonary arterial hypertension. J Am Coll Cardiol, 2004. 43: p. 40S$47 S$.

6. Souza R, CA, Pedra CA, Jardim C, Watge D, Campos FT, Meyer G, Arakaki JSO, Andrade LEC, Terra Filho M, Sampaio-Barros P, Maciel R, Menna-Barreto SS, Amado V, gomes WJ, Ditretrizes Brasileiras para o Manejo da Hipertensão Pulmonar. J Bras Pneumol, 2005. 31: p. S1-S31.

7. Kim, H, Yung, GL, Marsh, JJ, Konopka, RG, Pedersen, CA, Chiles, PG, Morris, TA, and Channick, RN, Endothelin mediates pulmonary 
vascular remodelling in a canine model of chronic embolic pulmonary hypertension. Eur Respir J, 2000. 15: p. 640-8.

8. MacLean, MR, Endothelin-1: a mediator of pulmonary hypertension? Pulm Pharmacol Ther, 1998. 11: p. 125-32.

9. Lapa, MS, Ferreira, EV, Jardim, C, Martins Bdo, C, Arakaki, JS, and Souza, R, [Clinical characteristics of pulmonary hypertension patients in two reference centers in the city of Sao Paulo]. Rev Assoc Med Bras, 2006. 52: p. 139-43.

10. Costa, EL, Jardim, C, Bogossian, HB, Amato, MB, Carvalho, CR, and Souza, R, Acute vasodilator test in pulmonary arterial hypertension: evaluation of two response criteria. Vascul Pharmacol, 2005. 43: p. 143-7.

11. Rich, S, Kaufmann, E, and Levy, PS, The effect of high doses of calcium-channel blockers on survival in primary pulmonary hypertension. N Engl J Med, 1992. 327: p. 76-81.

12. Barst, RJ, Rubin, LJ, Long, WA, McGoon, MD, Rich, S, Badesch, DB, Groves, BM, Tapson, VF, Bourge, RC, Brundage, BH, and et al., $A$ comparison of continuous intravenous epoprostenol (prostacyclin) with conventional therapy for primary pulmonary hypertension. The Primary Pulmonary Hypertension Study Group. N Engl J Med, 1996. 334: p. 296-302.

13. Hoeper, MM, Spiekerkoetter, E, Westerkamp, V, Gatzke, R, and Fabel, $\mathrm{H}$, Intravenous iloprost for treatment failure of aerosolised 
iloprost in pulmonary arterial hypertension. Eur Respir J, 2002. 20: p. 339-43.

14. Souza, R, Jardim, C, Martins, B, Cortopassi, F, Yaksic, M, Rabelo, R, and Bogossian, $\mathrm{H}$, Effect of bosentan treatment on surrogate markers in pulmonary arterial hypertension. Curr Med Res Opin, 2005. 21: p. 907-11.

15. Souza, R, Martins, BC, Jardim, C, Cortopassi, F, Fernandes, CJ, Pulido, T, and Sandoval, J, Effect of sitaxsentan treatment on quality of life in pulmonary arterial hypertension. Int J Clin Pract, 2007. 61: p. 153-6.

16. Silva, SMV, C. B. ; Bogossian, H. B. ; Jardim, Carlos ; Demarzo, S. E. ; Souza, R., Resolução do shunt direita-esquerda após o uso de sildenafil como tratamento para hipertensão pulmonar primária. Jornal de Pneumonologia - Sociedade Brasileira de Pneumologia e Tisiologia, 2003. 29: p. 1-4.

17. Carvalho, ACH, A L ; Fernandes, C J C ; Lapa, M ; Jardim, C ; Souza, R., Tadalafil as treatment for idiopathic pulmonary arterial hypertension. Arquivos Brasileiros de Cardiologia, 2007. 87: p. 195197.

18. Cohn, JN, Introduction to surrogate markers. Circulation, 2004. 109: p. IV20-1.

19. D'Alonzo, GE, Barst, RJ, Ayres, SM, Bergofsky, EH, Brundage, BH, Detre, KM, Fishman, AP, Goldring, RM, Groves, BM, Kernis, JT, and et al., Survival in patients with primary pulmonary hypertension. 
Results from a national prospective registry. Ann Intern Med, 1991. 115: p. 343-9.

20. Jardim, C, Rochitte, CE, Humbert, M, Rubenfeld, G, Jasinowodolinski, D, Carvalho, CR, and Souza, R, Pulmonary artery distensibility in pulmonary arterial hypertension: an MRI pilot study. Eur Respir J, 2007. 29: p. 476-81.

21. Michelakis, ED, Tymchak, W, Noga, M, Webster, L, Wu, XC, Lien, D, Wang, SH, Modry, D, and Archer, SL, Long-term treatment with oral sildenafil is safe and improves functional capacity and hemodynamics in patients with pulmonary arterial hypertension. Circulation, 2003. 108: p. 2066-9.

22. Miyamoto, S, Nagaya, N, Satoh, T, Kyotani, S, Sakamaki, F, Fujita, M, Nakanishi, N, and Miyatake, K, Clinical correlates and prognostic significance of six-minute walk test in patients with primary pulmonary hypertension. Comparison with cardiopulmonary exercise testing. Am J Respir Crit Care Med, 2000. 161: p. 487-92.

23. Leuchte, HH, Holzapfel, M, Baumgartner, RA, Ding, I, Neurohr, C, Vogeser, M, Kolbe, T, Schwaiblmair, M, and Behr, J, Clinical significance of brain natriuretic peptide in primary pulmonary hypertension. J Am Coll Cardiol, 2004. 43: p. 764-70.

24. Leuchte, HH, Holzapfel, M, Baumgartner, RA, Neurohr, C, Vogeser, $\mathrm{M}$, and Behr, J, Characterization of brain natriuretic peptide in longterm follow-up of pulmonary arterial hypertension. Chest, 2005. 128: p. 2368-74. 
25. Nagaya, N, Nishikimi, T, Uematsu, M, Satoh, T, Kyotani, S, Sakamaki, F, Kakishita, M, Fukushima, K, Okano, Y, Nakanishi, N, Miyatake, K, and Kangawa, K, Plasma brain natriuretic peptide as a prognostic indicator in patients with primary pulmonary hypertension. Circulation, 2000. 102: p. 865-70.

26. Souza, R, Bogossian, HB, Humbert, M, Jardim, C, Rabelo, R, Amato, $\mathrm{MB}$, and Carvalho, CR, N-terminal-pro-brain natriuretic peptide as a haemodynamic marker in idiopathic pulmonary arterial hypertension. Eur Respir J, 2005. 25: p. 509-13.

27. Souza, R, Jardim, C, Julio Cesar Fernandes, C, Silveira Lapa, M, Rabelo, R, and Humbert, M, NT-proBNP as a tool to stratify disease severity in pulmonary arterial hypertension. Respir Med, 2007. 101: p. 69-75.

28. Sitbon, O, Gressin, V, Speich, R, Macdonald, PS, Opravil, M, Cooper, DA, Fourme, T, Humbert, M, Delfraissy, JF, and Simonneau, G, Bosentan for the treatment of human immunodeficiency virusassociated pulmonary arterial hypertension. Am J Respir Crit Care Med, 2004. 170: p. 1212-7.

29. Paciocco, G, Martinez, FJ, Bossone, E, Pielsticker, E, Gillespie, B, and Rubenfire, M, Oxygen desaturation on the six-minute walk test and mortality in untreated primary pulmonary hypertension. Eur Respir J, 2001. 17: p. 647-52.

30. ATS, ATS statement: guidelines for the six-minute walk test. Am J Respir Crit Care Med, 2002. 166: p. 111-7. 
31. Hoeper, MM, Oudiz, RJ, Peacock, A, Tapson, VF, Haworth, SG, Frost, AE, and Torbicki, A, End points and clinical trial designs in pulmonary arterial hypertension: clinical and regulatory perspectives. J Am Coll Cardiol, 2004. 43: p. 48S-55S.

32. The World Health Organization Quality of Life assessment (WHOQOL): position paper from the World Health Organization. Soc Sci Med, 1995. 41: p. 1403-9.

33. Curtis, JR, Deyo, RA, and Hudson, LD, Pulmonary rehabilitation in chronic respiratory insufficiency. 7. Health-related quality of life among patients with chronic obstructive pulmonary disease. Thorax, 1994. 49: p. $162-70$.

34. McSweeny, AJ and Creer, TL, Health-related quality-of-life assessment in medical care. Dis Mon, 1995. 41: p. 1-71.

35. Jones, PW, Application of quality of life measures in chronic obstrutive pulmonary disease.4: p. 131-4.

36. McHorney, CA, Kosinski, M, and Ware, JE, Jr., Comparisons of the costs and quality of norms for the SF-36 health survey collected by mail versus telephone interview: results from a national survey. Med Care, 1994. 32: p. 551-67.

37. PASQUALI, LEV, Psicometria: Teoria dos testes na psicologia e na educação. 1 ed, ed. Vozes. Vol. 1. 2003, Petrópolis, RJ. 397.

38. McHorney, CA, Ware, JE, Jr., Lu, JF, and Sherbourne, CD, The MOS 36-item Short-Form Health Survey (SF-36): III. Tests of data quality, 
scaling assumptions, and reliability across diverse patient groups. Med Care, 1994. 32: p. 40-66.

39. Ciconelli RM, FM, Santos W, Meinão I, Quaresma MR, Tradução para a Língua Portuguesa e validação do questionário genérico da avaliação de qualidade de vida SF-36 (Brasil SF-36). Rev Bras Reumatol, 1999. 39: p. 143-50.

40. Jones, PW, Issues concerning health-related quality of life in COPD. Chest, 1995. 107: p. 187S-193S.

41. Wijkstra, PJ, TenVergert, EM, Van Altena, R, Otten, V, Postma, DS, Kraan, J, and Koeter, GH, Reliability and validity of the chronic respiratory questionnaire (CRQ). Thorax, 1994. 49: p. 465-7.

42. Sousa, TC, Jardim, JR, and Jones, P, Validação do Questionário do Hospital Saint George na doença respiratória (SGRQ) em pacientes portadores de doença pulmonar obstrutica crônica no Brasil. J. Pneumol, 2000. 26: p. 119-25.

43. Hajiro, T, Nishimura, K, Jones, PW, Tsukino, M, Ikeda, A, Koyama, H, and Izumi, T, A novel, short, and simple questionnaire to measure health-related quality of life in patients with chronic obstructive pulmonary disease. Am J Respir Crit Care Med, 1999. 159: p. 1874-8.

44. Ware, JE, Jr., What information do consumers want and how will they use it? Med Care, 1995. 33: p. JS25-30.

45. Mahler, DA and Mackowiak, JI, Evaluation of the short-form 36-item questionnaire to measure health-related quality of life in patients with COPD. Chest, 1995. 107: p. 1585-9. 
46. Ware, JE, Jr., Kosinski, M, Bayliss, MS, McHorney, CA, Rogers, WH, and Raczek, A, Comparison of methods for the scoring and statistical analysis of SF-36 health profile and summary measures: summary of results from the Medical Outcomes Study. Med Care, 1995. 33: p. AS264-79.

47. Ciconelli, R, Tradução para o português e validação do questionário genérico da avaliação de qualidade de vida "Medical Outcomes Study 36-Item Short-Form Health Survey (SF-36)". 1997, Escola Paulista de Medicina/ UNIFESP: São Paulo.

48. Gomberg-Maitland, M, Thenappan, T, Rizvi, K, Chandra, S, Meads, DM, and McKenna, SP, United States validation of the Cambridge Pulmonary Hypertension Outcome Review (CAMPHOR). J Heart Lung Transplant, 2008. 27: p. 124-30.

49. McKenna, SP, Doughty, N, Meads, DM, Doward, LC, and PepkeZaba, J, The Cambridge Pulmonary Hypertension Outcome Review (CAMPHOR): a measure of health-related quality of life and quality of life for patients with pulmonary hypertension. Qual Life Res, 2006. 15: p. 103-15.

50. Taichman, DB, Shin, J, Hud, L, Archer-Chicko, C, Kaplan, S, Sager, JS, Gallop, R, Christie, J, Hansen-Flaschen, J, and Palevsky, H, Health-related quality of life in patients with pulmonary arterial hypertension. Respir Res, 2005. 6: p. 92.

51. McLaughlin, VV, Presberg, KW, Doyle, RL, Abman, SH, McCrory, DC, Fortin, T, and Ahearn, G, Prognosis of pulmonary arterial 
hypertension: ACCP evidence-based clinical practice guidelines. Chest, 2004. 126: p. 78S-92S.

52. Borg, GA, Psychophysical bases of perceived exertion. Med Sci Sports Exerc, 1982. 14: p. 377-81.

53. Wilson, RC and Jones, PW, A comparison of the visual analogue scale and modified Borg scale for the measurement of dyspnoea during exercise. Clin Sci (Lond), 1989. 76: p. 277-82.

54. Cenedese, E, Speich, R, Dorschner, L, Ulrich, S, Maggiorini, M, Jenni, $\mathrm{R}$, and Fischler, M, Measurement of quality of life in pulmonary hypertension and its significance. Eur Respir J, 2006. 28: p. 808-15.

55. Cronbach, L, Coefficient alpha and the internal structures of tests. Psychometrika, 1951. 16: p. 297-334.

56. White, J, Hopkins, RO, Glissmeyer, EW, Kitterman, N, and Elliott, CG, Cognitive, emotional, and quality of life outcomes in patients with pulmonary arterial hypertension. Respir Res, 2006. 7: p. 55.

57. Shafazand, S, Goldstein, MK, Doyle, RL, Hlatky, MA, and Gould, MK, Health-related quality of life in patients with pulmonary arterial hypertension. Chest, 2004. 126: p. 1452-9.

58. Chua, R, Keogh, AM, Byth, K, and O'Loughlin, A, Comparison and validation of three measures of quality of life in patients with pulmonary hypertension. Intern Med J, 2006. 36: p. 705-10.

59. Peacock, A, Naeije, R, Galie, N, and Reeves, JT, End points in pulmonary arterial hypertension: the way forward. Eur Respir J, 2004. 23: p. 947-53. 
60. Hopman, WM, Towheed, T, Anastassiades, T, Tenenhouse, A, Poliquin, S, Berger, C, Joseph, L, Brown, JP, Murray, TM, Adachi, JD, Hanley, DA, and Papadimitropoulos, E, Canadian normative data for the SF-36 health survey. Canadian Multicentre Osteoporosis Study Research Group. CMAJ, 2000. 163: p. 265-71.

61. Senna, ER, Estudo sobre a prevalência de doenças reumáticas na cidade de Montes Claros, in Ciências da Saúde. 2002, Universidade Federal de São Paulo, UNIFESP, Brasil: São Paulo. 
Apêndices 


\section{APÊNDICE 1: QUESTIONÁRIO SF-36 PARA PESQUISA EM SAÚDE}

Instruções: Esta pesquisa questiona você sobre sua saúde. Estas informações nos manterão informados de como você se sente e quão bem você é capaz de fazer suas atividades de vida diária. Responda cada questão marcando a resposta como indicado. Caso você esteja inseguro em como responder, por favor, tente responder o melhor que puder.

\section{1 - Em geral você diria que sua saúde é:}

(Circule uma)

Excelente

2 - Comparada há 1 ano atrás, como você classificaria sua saúde em geral, agora?

(Circule uma)

Muito melhor agora do que há um ano atrás ............................................. 1

Um pouco melhor agora do que há um ano atrás ....................................... 2

Quase a mesma de um ano atrás .................................................................. 3

Um pouco pior agora do que há um ano atrás ............................................ 4

Muito pior agora do que há um ano atrás ................................................. 5 
3 - Os seguintes itens são sobre atividades que você poderia fazer atualmente durante um dia comum. Devido a sua saúde, você tem dificuldade para fazer essas atividades? Neste caso, quanto?

(circule um número em cada linha)

\begin{tabular}{|l|c|c|c|}
\hline \multicolumn{1}{|c|}{ Atividades } & $\begin{array}{c}\text { Sim } \\
\text { dificulta } \\
\text { muito }\end{array}$ & $\begin{array}{c}\text { Sim } \\
\text { dificulta um } \\
\text { pouco }\end{array}$ & $\begin{array}{c}\text { Não. Não } \\
\text { dificulta de } \\
\text { modo algum }\end{array}$ \\
\hline $\begin{array}{l}\text { a - Atividades vigorosas, que exigem muito } \\
\text { esforço, tais como: correr, levantar objetos } \\
\text { pesados, participar em esportes árduos. }\end{array}$ & 1 & 2 & 3 \\
\hline $\begin{array}{l}\text { b - Atividades moderadas, tais como: mover uma } \\
\text { mesa, passar aspirador de pó, jogar bola, varrer a } \\
\text { casa. }\end{array}$ & 1 & 2 & 3 \\
\hline \hline c - Levantar ou carregar mantimentos & 1 & 2 & 3 \\
\hline \hline d - Subir vários lances de escada & 1 & 2 & 3 \\
\hline \hline e- Subir um lance de escada & 1 & 2 & 3 \\
\hline f - Curvar-se, ajoelhar-se ou dobrar-se & 1 & 2 & 3 \\
\hline \hline - Andar mais de 1 quilômetro & 1 & 2 & 3 \\
\hline \hline h- Andar vários quarteirões & 1 & 2 & 3 \\
\hline \hline i- Andar um quarteirão & 1 & 3 \\
\hline \hline j- Tomar banho ou vestir-se & 1 & 2 & 3 \\
\hline
\end{tabular}


4 - Durante as últimas 4 semanas,você teve algum dos seguintes problemas com o seu trabalho ou com alguma atividade diária regular, como consequiência de sua saúde física?

(circule um número em cada linha)

\begin{tabular}{|l|c|c|}
\hline & Sim & Não \\
\hline \begin{tabular}{l|c|c|}
\hline a - Você diminuiu a quantidade de tempo que se dedicava ao seu \\
trabalho ou a outras atividades?
\end{tabular} & 1 & 2 \\
\hline \begin{tabular}{|l|c|c|}
\hline b- Realizou menos tarefas do que você gostaria? & 1 & 2 \\
\hline c-Esteve limitado no seu trabalho ou em outras atividades? & 1 & 2 \\
\hline $\begin{array}{l}\text { d- Teve dificuldade de fazer seu trabalho ou outras atividades? } \\
\text { (necessitou de um esforço extra?) }\end{array}$ & 1 & 2 \\
\hline
\end{tabular}
\end{tabular}

5 - Durante as últimas 4 semanas, você teve algum dos seguintes problemas com o seu trabalho ou outra atividade regular diária,como conseqüência de algum problema emocional (como sentir-se deprimido ou ansioso)?

(circule um número em cada linha)

\begin{tabular}{|c|c|c|}
\hline & Sim & Não \\
\hline $\begin{array}{l}\text { a - Você diminuiu a quantidade de tempo que se dedicava ao seu } \\
\text { trabalho ou a outras atividades? }\end{array}$ & 1 & 2 \\
\hline b- Realizou menos tarefas do que você gostaria? & 1 & 2 \\
\hline $\begin{array}{l}\text { c - Não trabalhou ou não fez qualquer das atividades com tanto } \\
\text { cuidado como geralmente faz? }\end{array}$ & 1 & 2 \\
\hline
\end{tabular}


6 - Durante as últimas 4 semanas, de que maneira sua saúde física ou problemas emocionais interferiram nas suas atividades sociais normais, em relação a família, vizinhos, amigos ou em grupo?

(Circule uma)

De forma nenhuma

7 - Quanta dor no corpo você teve durante as últimas 4 semanas?

(Circule uma)

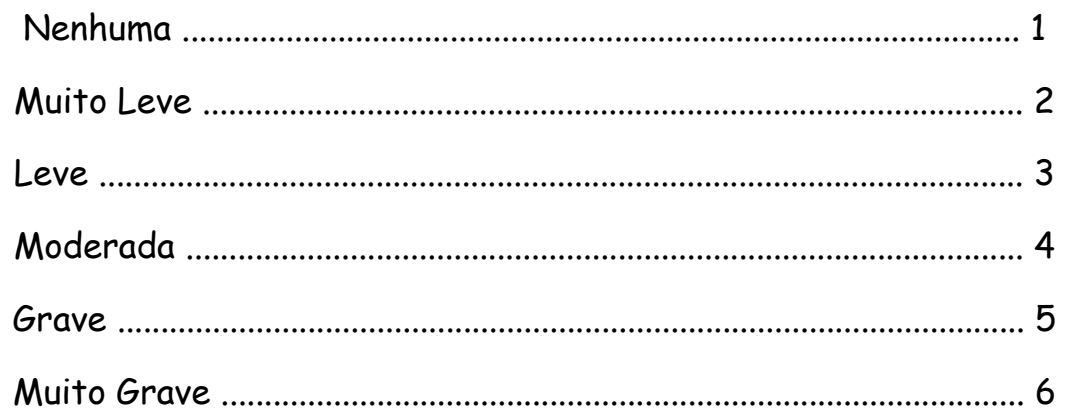

8 - Durante as últimas 4 semanas, quanto a dor interferiu com o seu trabalho normal (incluindo tanto o trabalho, fora de casa e dentro de casa)?

(Circule uma)

De maneira alguma
Um pouco
Moderadamente
Bastante
Extremamente


9 - Estas questões são sobre como você se sente e como tudo tem acontecido com você durante as últimas 4 semanas. Para cada questão, por favor dê uma resposta que mais se aproxime da maneira como você se sente. Em relação as últimas 4 semanas.

(Circule um número para cada linha)

\begin{tabular}{|c|c|c|c|c|c|c|}
\hline & $\begin{array}{c}\text { Todo } \\
\text { tempo }\end{array}$ & $\begin{array}{c}\text { A maior } \\
\text { parte } \\
\text { do } \\
\text { tempo }\end{array}$ & $\begin{array}{c}\text { Uma } \\
\text { boa } \\
\text { parte } \\
\text { do } \\
\text { tempo }\end{array}$ & $\begin{array}{c}\text { Alguma } \\
\text { parte } \\
\text { do } \\
\text { tempo }\end{array}$ & $\begin{array}{c}\text { Uma } \\
\text { pequena } \\
\text { parte } \\
\text { do } \\
\text { tempo }\end{array}$ & Nunca \\
\hline
\end{tabular}

a - Quanto tempo você tem se sentido cheio de vigor, cheio de vontade, cheio de força?

\begin{tabular}{|l|l|l|}
1 & \\
& &
\end{tabular}

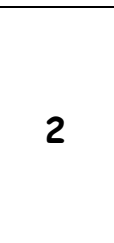$$
\text { (1) }
$$

(1)

\begin{tabular}{|l|l|l|l|}
\hline 3 & 4 & 5 & 6 \\
\hline
\end{tabular}

\begin{tabular}{|l|l|l|l|l|l|l|}
\hline $\begin{array}{l}\mathrm{b}-\text { Quanto tempo você tem se } \\
\text { sentido uma pessoa muito } \\
\text { nervosa? }\end{array}$ & 1 & 2 & 3 & 4 & 5 & 6 \\
\hline
\end{tabular}

\begin{tabular}{|l|l|l|l|l|l|l|}
\hline $\begin{array}{l}\text { c - Quanto tempo você tem se } \\
\text { sentido tão deprimido que nada } \\
\text { pode animá-lo? }\end{array}$ & 1 & 2 & 3 & 4 & 5 & 6 \\
\hline
\end{tabular}

\begin{tabular}{|l|l|l|l|l|l|l|}
\hline $\begin{array}{l}d \text { - Quanto tempo você tem se } \\
\text { sentido calmo ou tranqüilo? }\end{array}$ & 1 & 2 & 3 & 4 & 5 & 6 \\
\hline
\end{tabular}

\begin{tabular}{|l|l|l|l|l|l|l|}
\hline $\begin{array}{l}\text { e - Quanto tempo você tem se } \\
\text { sentido com muita energia? }\end{array}$ & 1 & 2 & 3 & 4 & 5 & 6 \\
\hline
\end{tabular}

\begin{tabular}{|l|l|l|l|l|l|l|}
\hline $\begin{array}{l}\text { f - Quanto tempo você tem se } \\
\text { sentido desanimado e abatido? }\end{array}$ & 1 & 2 & 3 & 4 & 5 & 6 \\
\hline
\end{tabular}

\begin{tabular}{|l|l|l|l|l|l|l|}
\hline $\begin{array}{l}\text { 9- Quanto tempo você tem se } \\
\text { sentido esgotado? }\end{array}$ & 1 & 2 & 3 & 4 & 5 & 6 \\
\hline
\end{tabular}

\begin{tabular}{|l|l|l|l|l|l|l|}
\hline $\begin{array}{l}\text { h- Quanto tempo você tem se } \\
\text { sentido uma pessoa feliz? }\end{array}$ & 1 & 2 & 3 & 4 & 5 & 6 \\
\hline $\begin{array}{l}\text { I - Quanto tempo você tem se } \\
\text { sentido cansado? }\end{array}$ & 1 & 2 & 3 & 4 & 5 & 6 \\
\hline
\end{tabular}


10 - Durante as últimas 4 semanas, quanto do seu tempo a sua saúde física ou problemas emocionais interferiram com as suas atividades sociais (como visitar amigos, parentes, etc.)?

(Circule uma)

Todo o tempo 1

A maior parte do tempo 2

Alguma parte do tempo 3

Uma pequena parte do tempo 4

Nenhuma parte do tempo 5

11 - O quanto verdadeiro ou falso é cada uma das afirmações

(Circule um número me cada linha)

\begin{tabular}{|l|c|c|c|c|c|}
\hline & $\begin{array}{c}\text { Definitiva } \\
\text { mente } \\
\text { verdadeir } \\
\text { o }\end{array}$ & $\begin{array}{c}\text { A maioria } \\
\text { das vezes } \\
\text { verdadeir } \\
\text { a }\end{array}$ & Não sei & $\begin{array}{c}\text { A maioria } \\
\text { das vezes } \\
\text { falsa }\end{array}$ & $\begin{array}{c}\text { Definitiva } \\
\text { mente } \\
\text { falsa }\end{array}$ \\
\hline $\begin{array}{l}\text { a - Eu costumo adoecer um pouco mais } \\
\text { facilmente que as outras pessoas }\end{array}$ & 1 & 2 & 3 & 4 & 5 \\
\hline $\begin{array}{l}\text { b - Eu sou tão saudável quanto qualquer } \\
\text { pessoa que eu conheço. }\end{array}$ & 1 & 2 & 3 & 4 & 5 \\
\hline c-Eu acho que a minha saúde vai piorar & 1 & 2 & 3 & 4 & 5 \\
\hline d- Minha saúde é excelente & 1 & 2 & 3 & 4 & 5 \\
\hline
\end{tabular}




\section{APÊNDICE 1.1 : PONTUAÇÃO DO QUESTIONÁRIO SF-36}

\section{QUESTÃo}

$1 \quad 1=5,0$

2

3

4

5

6

$5=1$

$7 \quad 1=6,0$

$6=1,0$

8

Se $8=1$ e $7=1=6$

Se $8=1$ e $7=2$ a $6=5$

Se $8=2$ e $7=2$ a $6=4$

Se $8=4$ e $7=2$ a $6=2$

Se $8=5$ e $7=2$ a $6=1$

\section{PONTUAÇÃO}

$3=3,4$

$4=2,0$

$5=1,0$

Soma Normal

Soma Normal

Soma Normal

Soma Normal

$2=4$

$3=3$

$4=2$

$3=4,2$

$4=3,1$

$5=2,2$

Se a questão 7 não for respondida, o escore da questão 8

passa a ser o seguinte:

$$
\begin{aligned}
& 1=6,0 \\
& 2=4,75 \\
& 3=3,5 \\
& 4=2,25 \\
& 5=1,0
\end{aligned}
$$

Se $8=3$ e $7=2$ a $6=3$

9 a,d,e,h $=$ valores contrários:

$(1=6 ; 2=5 ; 3=4 ; 4=3 ; 5=2 ; 6=1)$

Vitalidade $=\mathrm{a}+\mathrm{e}+\mathrm{g}+\mathrm{i}$

Saúde Mental $=\mathrm{b}+\mathrm{c}+\mathrm{d}+\mathrm{f}+\mathrm{h}$

11 a,c $=$ valores normais

b,d $=$ valores contrários $(1=5 ; 2=4 ; 3=3 ; 4=2 ; 5=1)$ 
APÊNDICE 1.2 : CÁLCULO DOS ESCORES PARA O QUESTIONÁRIO SF-36

Escala Bruta (Raw Scale) [0 - 100]

Variação Dos Escores

\section{Questão Limites}

Capacidade

Funcional

Aspectos Físicos

Dor

\section{Estado Geral \\ de Saúde}

Vitalidade

Aspectos Sociais

Aspecto

Emocional

Saúde Mental

$$
\begin{gathered}
3 \\
(a+b+c+d+e+f+g+h+i+j)
\end{gathered}
$$

10,30

20

4

$$
(a+b+c+d)
$$

4, 8

4

$7+8$

2, 12

10

$1+11$

5, 25

20

9

4, 24

20

$$
(a+e+g+i)
$$

$$
6+10
$$

2, 10

8

5

3, 6

3

$(a+b+c)$

9

5,30

25

$$
(b+c+d+f+h)
$$

\section{Escala Bruta:}

ltem $=[$ Valor obtido - Valor mais baixo $] \times 100$

$$
\text { Variação }
$$

Exemplo:

Capacidade funcional $=21$

Valor mais baixo $=10$

Cálculo $=\underline{21-10} \times 100=55$

Variação $=20$

20

A questão número 2 não entra no cálculo dos domínios

Dados perdidos: Se responder mais de 50\% = substituir o valor pela média. 